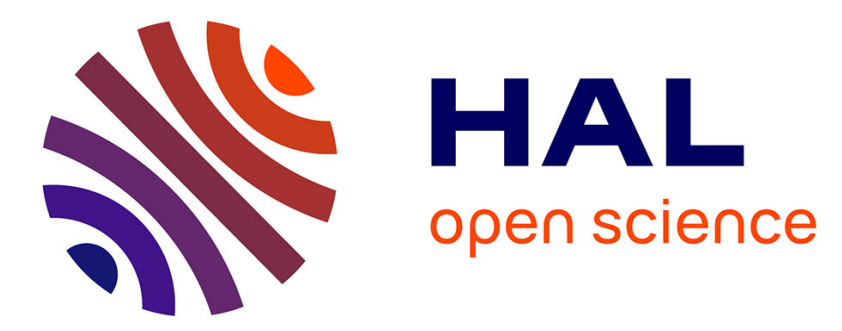

\title{
Elastic properties of fractured rock masses with frictional properties and power-law fracture size distributions
}

\author{
Philippe Davy, Caroline Darcel, Romain Le Goc, Diego Mas Ivars
}

\section{To cite this version:}

Philippe Davy, Caroline Darcel, Romain Le Goc, Diego Mas Ivars. Elastic properties of fractured rock masses with frictional properties and power-law fracture size distributions. Journal of Geophysical Research : Solid Earth, 2018, 123 (8), pp.6521-6539. 10.1029/2017JB015329 . insu-01857500

HAL Id: insu-01857500

https://hal-insu.archives-ouvertes.fr/insu-01857500

Submitted on 18 Oct 2018

HAL is a multi-disciplinary open access archive for the deposit and dissemination of scientific research documents, whether they are published or not. The documents may come from teaching and research institutions in France or abroad, or from public or private research centers.
L'archive ouverte pluridisciplinaire HAL, est destinée au dépôt et à la diffusion de documents scientifiques de niveau recherche, publiés ou non, émanant des établissements d'enseignement et de recherche français ou étrangers, des laboratoires publics ou privés. 


\section{Journal of Geophysical Research: Solid Earth}

\section{RESEARCH ARTICLE}

10.1029/2017JB015329

\section{Special Section: \\ Rock Physics of the Upper \\ Crust}

\section{Key Points:}

- Elastic properties are derived for rock masses that contain frictional fractures defined by an elastic stiffness and a strength limit

- Analytical solutions of the Young's modulus and Poisson's ratio are given for a population of fractures embedded in an elastic matrix

- The ratio between matrix and fracture stiffness is a length that controls the elastic properties for power law distributed fracture sizes

Correspondence to:

P. Davy,

philippe.davy@univ-rennes1.fr

Citation:

Davy, P., Darcel, C., Le Goc, R., \& Mas Ivars, D. (2018). Elastic properties of fractured rock masses with frictional properties and power law fracture size distributions. Journal of Geophysical Research: Solid Earth, 123, 6521-6539. https://doi.org/10.1029/2017JB015329

Received 7 DEC 2017 Accepted 10 JUL 2018

Accepted article online 16 JUL 2018

Published online 25 AUG 2018

\section{Elastic Properties of Fractured Rock Masses With Frictional Properties and Power Law Fracture Size Distributions}

\author{
P. Davy' ${ }^{1 D}$, C. Darcel², R. Le Goc ${ }^{2}$ (D) and D. Mas Ivars ${ }^{3,4}$ iD \\ ${ }^{1}$ Université Rennes, CNRS, Géosciences Rennes, UMR 6118, Rennes, France, ${ }^{2}$ Itasca Consultants SAS, Écully, France, \\ ${ }^{3}$ Swedish Nuclear Fuel and Waste Management Company, SKB, Solna, Sweden, ${ }^{4}$ KTH Royal Institute of Technology, \\ Stockholm, Sweden
}

Abstract We derive the relationships that link the general elastic properties of rock masses to the geometrical properties of fracture networks, with a special emphasis to the case of frictional crack surfaces. We extend the well-known elastic solutions for free-slipping cracks to fractures whose plane resistance is defined by an elastic fracture (shear) stiffness $k_{s}$ and a stick-slip Coulomb threshold. A complete set of analytical solutions have been derived for (i) the shear displacement in the fracture plane for stresses below the slip threshold and above, (ii) the partitioning between the resistances of the fracture plane on the one hand and of the elastic matrix on the other hand, and (iii) the stress conditions to trigger slip. All the expressions have been checked with numerical simulations. The Young's modulus and Poisson's ratio were also derived for a population of fractures. They are controlled both by the total fracture surface for fractures larger than the stiffness length $l_{S}$ (defined by $k_{s}$ and the intact matrix elastic properties) and by the percolation parameter of smaller fractures. These results were applied to power law fracture size distributions, which are likely relevant to geological cases. We show that if the fracture size exponent is in the range -3 to -4 , which corresponds to a wide range of geological fracture networks, the elastic properties of the bulk rock are almost exclusively controlled by $k_{s}$ and the stiffness length, meaning that the fractures of size $I_{S}$ play a major role in the definition of the elastic properties.

\section{Introduction}

Assessing fractured rock mass effective mechanical properties is a prerequisite to many geotechnical applications and a still major scientific issue about the way to take account of heterogeneities of the rock mass. Among all potential heterogeneities, fractures are those whose impact on rock strength and stiffness is prevalent (Amadei \& Goodman, 1981; Barton et al., 1974; Bieniawski, 1973, 1978; Hoek, 1994; Hoek \& Diederichs, 2006; Hoek et al., 1995; Singh, 1973), with the difficulty that the distribution of fractures is complex with a wide range of spatial scales involved; the density can be highly variable in space, and the geometrical and statistical models of fracture patterns are still debated.

The importance of fractures in altering the effective properties of a rock was noted by Simmons and Brace (1965) and Walsh (1965a). The quantitative analysis of these expected consequences is well established for simple cases, as a single frictionless disk crack embedded in a homogeneous elastic rock matrix (see Fabrikant, 1990; Sneddon \& Lowengrub, 1969, for detailed mathematics or the review by Atkinson, 1987), but the properties of rock masses with a complex set of fractures are still an issue. Expressions have been derived for networks of frictionless cracks with limited size range by neglecting or simplifying stress interactions (see review in Grechka \& Kachanov, 2006; Guéguen \& Kachanov, 2011; Kachanov, 1993; Sayers \& Kachanov, 1995; Schoenberg \& Sayers, 1995). The main controlling factor is the percolation parameter of the fracture network (the definitions are given in the next section), which is a volumetric measure (the sum of sphere volumes around cracks divided by the total volume) that also controls network connectivity (de Dreuzy et al., 2000).

The application of these theories to actual rock masses raises two main issues. The first is about the role of frictional stresses in the damaged elastic modulus, since friction is likely prevailing on large geological fractures. Friction was introduced in the damaged elastic models as a force independent of the displacement, generally defined by a Coulomb law (Gambarotta \& Lagomarsino, 1993; Halm \& Dragon, 1998; Horii \& Nemat-Nasser, 1983; Kachanov, 1982; Walsh, 1965b, 1965c, among others) or as a series of elastic contacts between fracture walls (Kachanov et al., 2010; Sevostianov \& Kachanov, 2008a, 2008b, 2009; 
Yoshioka \& Scholz, 1989a, 1989b). This is even an issue for open cracks, where near-tip contact zones have consequences on the general repartition of stresses as well as on the stress intensity factors that describe the concentration of stress at the crack tips and control potential crack growth (Audoly, 2000; Comninou, 1977; Comninou \& Dundurs, 1980; Rice, 1988). In the geomechanical or geophysical literature, the complexity of contact processes that make friction is generally lumped into a few relationship and constitutive parameters of which the fracture stiffness, either normal or shear, relates stress and displacement at the fracture walls (Bandis et al., 1983; Goodman et al., 1968; Yoshioka \& Scholz, 1989a, 1989b) and the Coulomb criterion marks the limit between friction stick (elastic deformation) and slip (Byerlee \& Brace, 1968).

A second issue relevant to actual rocks is the intrinsic complexity of fracture networks, which results in a wide range of fracture sizes from micrometer to kilometer scales (Bonnet et al., 2001). This raises key questions about the integration of this large density distribution on the mechanical properties and on the critical scales that control them.

In this paper, we aim to discuss the relationship between a discrete fracture network (DFN) description of fractured rock masses (Davy et al., 2013; Dershowitz \& Einstein, 1988; Elmo et al., 2014; Jing et al., 2007; Long et al., 1982; Painter \& Cvetkovic, 2005; Selroos et al., 2002) with their effective elastic properties (mainly Young' modulus and Poisson's ratio). We develop the case, where the matrix surrounding fractures is homogeneous, and the friction on fracture walls is defined by constant shear and normal stiffnesses in the fracture plane with a stress threshold. Although very simple in the description of friction processes, this model allows us (i) to derive simple analytical expressions for the elastic parameters based on measurable parameters and (ii) to discuss the scaling and critical scales of rock mass properties for the cases of fracture network with a large range (power law) size distribution.

The paper is organized as follows: We first present the analytical equations of a single frictional fracture embedded in an elastic intact rock, we derive the expressions for a population of fractures, we discuss the relationships between fracture network densities and elastic properties, and finally, we derive the consequences of these concepts to geologically relevant fracture size distributions.

\section{Fracture Embedded in an Elastic Intact Rock}

Although very simple and commonly described in geomechanics textbooks, we develop the expression of the stress, strain, and fracture displacement of a disk-shaped fracture embedded in an elastic medium. We generalize the classical expression originally developed for a freely slipping crack to fractures, whose surface is resisting with an elastic shear stiffness $k_{s}$.

\subsection{Generalization of the Free-Slip Relationship for Frictional Cracks}

The shear and normal displacements, $t$ and $u$, respectively, are well known for a freely slipping disk fracture of diameter $I_{f}$, embedded in an infinite elastic rock matrix (i.e., intact rock) with Young's modulus $E_{m}$ and Poisson's ratio $v_{m}$ (e.g., Kachanov \& Sevostianov, 2013; Sneddon \& Lowengrub, 1969; the subscripts $m$ and $f$ refer to matrix and fracture properties, respectively)

$$
\begin{gathered}
t(r)=\frac{4\left(1-v_{m}^{2}\right)}{\pi\left(1-\frac{v_{m}}{2}\right)} \tau_{m} \frac{I_{f}}{E_{m}} \sqrt{1-\left(\frac{2 r}{I_{f}}\right)^{2}} \\
u=\frac{4\left(1-v_{m}^{2}\right)}{\pi} \frac{\min \left(\sigma_{m}, 0\right) I_{f}}{E_{m}} \sqrt{1-\left(\frac{2 r}{I_{f}}\right)^{2}}
\end{gathered}
$$

where $r$ is the distance to the disk center. Since these equations are established for freely slipping fractures, the stresses $\tau_{m}$ and $\sigma_{m}$ are the shear and normal stresses (respectively) due to the matrix deformation only (with the convention that normal stress and displacement are positive in compression). The average displacement, $\bar{t}$, calculated by integrating equation (1) over the fracture plane, is two thirds the displacement at the 


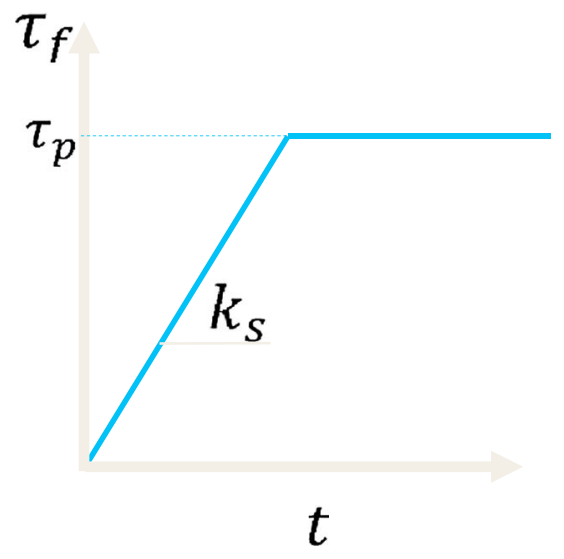

Figure 1. Rheological model of fracture slip: Relationship between the shear stress acting on the fracture plane $\tau_{f}$ and the relative displacement between the fracture walls $t$. The stress threshold $\tau_{p}$ marks the limit between an elastic behavior characterized by a fracture stiffness $k_{s}$, and a regime of constant friction $\tau_{f}=\tau_{p}$ fracture center, which leads to an apparent elastic shear stiffness of the matrix surrounding the fracture, $k_{m}$, such as

$$
k_{m}=\frac{\tau_{m}}{\bar{t}}=\frac{3 \pi}{8} \frac{1-\frac{v_{m}}{2}}{1-v_{m}^{2}} \frac{E_{m}}{l_{f}}
$$

$k_{m}$ will be called the matrix-fracture stiffness thereafter; to simplify the equation writing, it may also be written in the following as

$$
k_{m}=\frac{E_{m}^{*}}{l_{f}} E_{m}^{*}=\frac{3 \pi}{8} \frac{1-\frac{v_{m}}{2}}{1-v_{m}^{2}} E_{m}
$$

A similar expression can be derived for the normal stress and displacement, which will be discussed later in this section.

We generalize these expressions for nonfreely slipping fractures, that is, when there exists a resistance between both fracture walls that can be either elastic or frictional (i.e., a friction stress). This problem was partly addressed by Glubokovskikh et al. (2016) and Kachanov et al. (2010) for a set of asperity contacts between fracture walls. In this study, we consider a simplified elastic-plastic behavior, where the elastic resistance of fracture walls is modeled by constant elastic stiffness coefficients, $k_{s}$ for shear and $k_{n}$ for normal displacement, which are supposed constant over the whole fracture surface. A plastic limit $\tau_{p}$ is defined for shear displacement, above which the fracture freely slips, that is, the displacement $t$ becomes independent of $\tau$ (see, e.g., experimental data in Grasselli \& Egger, 2003; Figure 1):

$$
\tau_{f}=\min \left(k_{s} t, \tau_{p}\right)
$$

The shear displacement along the fracture plane is supposed to be 10 to $>1,000$ larger than the normal one (Bandis et al., 1983; Yoshinaka \& Yamabe, 1986) in compression. Depending on the stress intensity and on the fracture spacing, the normal displacement can be equivalent or even larger than the matrix deformation; thus, $k_{n}$ must be considered if the stress and fracture orientations preclude shearing. We discuss in detail the shear displacement in the following lines and briefly the normal displacement in section 2.6.

The remote stress $\tau$ is partitioned into two terms: (i) the resistance to shear displacement across fracture walls $\tau_{f}$ and (ii) the elastic stress generated by matrix deformation $\tau_{m}=k_{m} \bar{t}$ (equation (3)).

In the case where the fracture resistance is elastic, the eventual result consists in summing both stresses in the fracture and in the matrix, while the displacement of the former induces the deformation of the latter. For this configuration (same displacement, additional stress), we expect the total system stiffness to be the sum of fracture and matrix-fracture stiffnesses as it is done for asperity contacts (Barber, 2003; Kachanov et al., 2010; Sevostianov \& Kachanov, 2008a, 2008b, 2009). For a simple shear applied to the fracture surface, we expect the displacement $t(r)$ along the fracture walls to be written as follows:

$$
\frac{\tau}{t(r)}=\frac{\frac{2}{3} k_{m}}{\sqrt{1-\left(\frac{2 r}{l_{f}}\right)^{2}}}+k_{s}
$$

If $\tau_{f}=\tau_{p}$, the above equation must be rewritten as follows:

$$
\frac{\tau-\tau_{p}}{t(r)}=\frac{\frac{2}{3} k_{m}}{\sqrt{1-\left(\frac{2 r}{l_{f}}\right)^{2}}}
$$



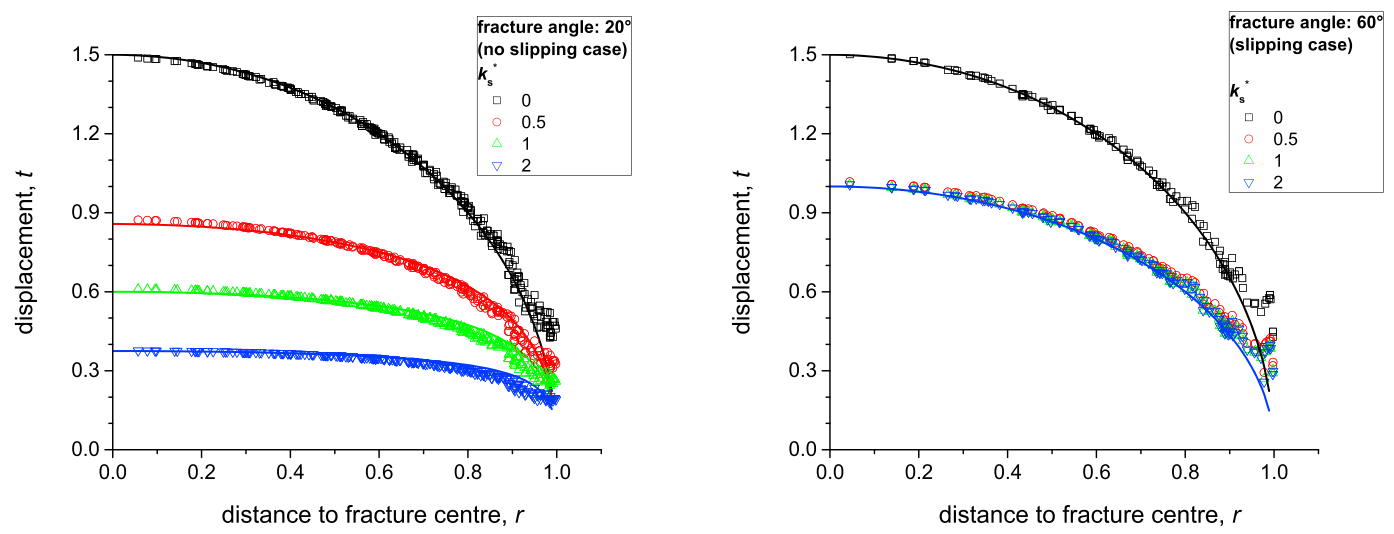

Figure 2. Displacement along the fracture plane calculated for a fracture embedded in an elastic matrix for four different values of $k_{s}^{*}=k_{s} / k_{m}: 0$ (black), 0.5 (red), 1 (green), and 2 (blue). The symbols are values obtained from 3DEC's calculations; the full lines are analytical expressions (equations (6) and (7)). A compressive stress is applied on one axis with no pressure on the other two axes, and the friction-slip condition is a linear Mohr-Coulomb relationship with a friction angle of $30^{\circ}$ and no cohesion: $\tau_{p}=\tan 30^{\circ} \cdot \sigma_{n}$. Left graph: No-slipping case $\left(\tau<\tau_{p}\right)$ obtained with an angle between the fracture normal and the compressive stress axis of $20^{\circ}$. Right: Slipping case $\left(\tau>\tau_{p}\right)$, obtained with an angle of $60^{\circ}$; the red, green, and blue symbols are almost overlapping for this case. For both graphs, displacements are normalized by the ratio $\frac{\tau}{k_{m}}$, and the fits are obtained by using equations (6); left) and (7); right).

Both expressions have been verified by numerical simulations using the 3-D distinct element code 3DEC@ (Israelsson, 1996; Itasca Consulting Group, 2016; Figure 2).

The average displacement relationship can be formally deduced from integrating equations (6) and (7) over the fracture plane:

$$
\bar{t}=\frac{1}{\pi R^{2}} \int_{\theta} \int_{0}^{R} t(r) r \mathrm{~d} r \mathrm{~d} \theta=\frac{2}{R^{2}} \int_{0}^{R} t(r) r \mathrm{~d} r
$$

where $R$ is the fracture radius, $R=\frac{l_{f}}{2}$. The case, where the fracture is slipping (equation (7)), is similar to the free-slipping case and can be easily integrated as follows:

$$
\bar{t}=\frac{\tau-\tau_{p}}{k_{m}}
$$

If the fracture is not slipping, the integral combines equations (6) and (8):

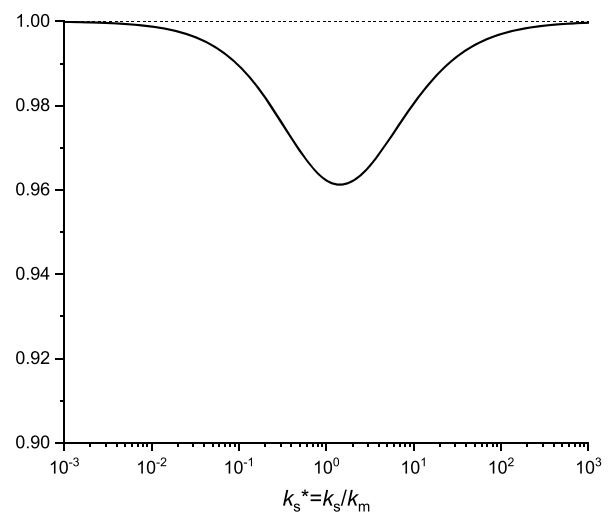

Figure 3. Plot of the ratio between equations (11) and (12). The latter gives an approximate solution of the average displacement in the fracture plane, which is accurate to within $4 \%$ compared to the exact solution.

$$
\bar{t}=\frac{\tau}{k_{m}} \frac{2}{R^{2}} \int_{0}^{R} \frac{r \mathrm{~d} r}{\frac{2 / 3}{\sqrt{1-\left(\frac{r}{R}\right)^{2}}}+k_{s}^{*}}
$$

with $k_{s}^{*}=\frac{k_{s}}{k_{m}}$.

By substituting $r$ by $u=1+\frac{3}{2} k_{s}^{*} \sqrt{1-\left(\frac{r}{R}\right)^{2}}$, we obtain

$$
\bar{t}=\frac{\tau}{k_{m}} \int_{1}^{1+\frac{3}{2} k_{s}^{*}} \frac{(u-1)^{2}}{\left(\frac{3}{2} k_{s}^{*}\right)^{3} u} \mathrm{~d} u=\frac{\tau}{k_{s}}\left(1-\frac{4}{3} \frac{1}{k_{s}^{*}}+\frac{8}{9} \frac{\ln \left(1+\frac{3}{2} k_{s}^{*}\right)}{k_{s}^{*} 2}\right)
$$

Figure 3 shows that a reasonable approximation of equation (11) is given by the simple expression:

$$
\bar{t}=\frac{\tau}{k_{m}+k_{s}}
$$



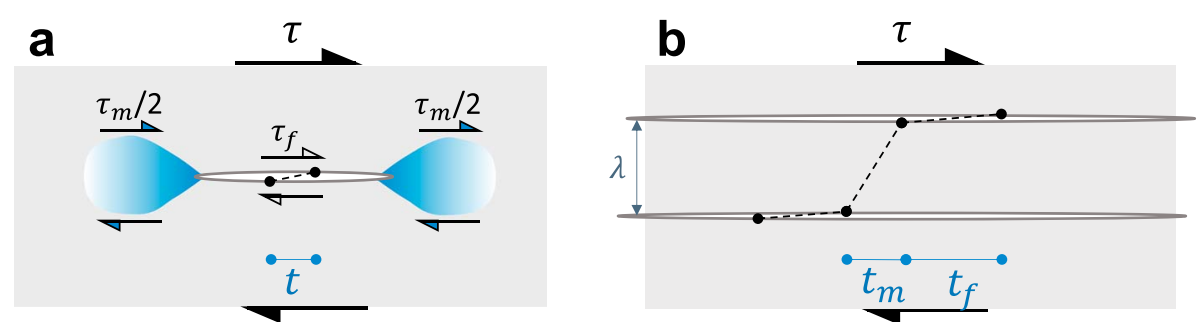

Figure 4. Difference between the model of a crack embedded in an elastic rock matrix that we treat in this paper (a) and the infinite crack model (Griffith et al., 2009). In the former, (a) the same displacement $t$ applies to both fracture and matrix while fracture and matrix stresses are additive $\left(\tau=\tau_{f}+\tau_{m}\right)$; in the latter, (b) the same shear stress $\tau$ applies to fracture and matrix, while the total displacement is the sum of that of fracture and matrix $\left(t=t_{f}+t_{m}\right)$.

Equation (12) is valid for both end-members $k_{m} \gg k_{s}$ and $k_{m} \ll k_{s}$. For any value of $k_{s}$, the difference between equations (11) and (12) is less than $4 \%$ (Figure 3).

Equations (9) and (12) actually quantify the stress partitioning between the average resistance of the fracture plane $\overline{\tau_{f}}$ on the one hand and the resistance to deformation of the surrounding matrix on the other hand $\overline{\tau_{m}}$.

$$
\tau=\overline{\tau_{f}}+\overline{\tau_{m}}=\min \left(k_{s} \bar{t}, \tau_{p}\right)+k_{m} \bar{t}
$$

This result calls for several remarks that we develop below.

\subsection{Difference With Other Models With Elastic Matrix and Fracture Stiffness}

The expression is different from the infinite-fracture model, such as theorized by Griffith et al. (2009; see Figure 4). In the infinite-fracture case, the same stress applies to both fractures and matrix in between fractures, but the deformation of the matrix $\epsilon_{m}$ and the displacement of fracture walls $t_{f}$ are independent; the former depends on the matrix shear modulus $G_{m}$ and the latter on $k_{s}$. In the case of a fracture embedded in an infinite medium, $t_{f}$ is both the displacement of fracture walls and the cause of matrix deformation $\epsilon_{m}$ so that $t_{f}$ applies to both $\tau_{f}$ and $\tau_{m}$ but both stresses are independent.

Both models lead to a stress-displacement relationship, which combines the fracture $\left(k_{s}\right)$ and matrix $\left(E_{m}\right.$ or $G_{m}$ ) properties with a characteristic length scale $\lambda$ that is required to compare stiffness and elastic modulus. The expression can be written in a general way as: $t=\frac{\tau}{\text { average }\left(k_{s}, \frac{E_{m} \text { or } G_{m}}{\lambda}\right)}$, where average() is an averaging function, which differs from one model to the other. In the infinite-fracture model, the displacement is the harmonic average of the shear modulus $G_{m}$ and fracture stiffness $k_{s}$, and $\lambda$ is equal to the fracture spacing $t=\tau *\left(\frac{\lambda}{G_{m}}+\frac{1}{k_{s}}\right)$. In contrast, the fracture-in-matrix model leads to an arithmetic average of $E_{m}$ and $k_{s}$, with a characteristic length equal to the fracture length.

This leads to very different predictions that can be highlighted by the example of an infinitely rigid matrix $\left(G_{m}, E_{m} \gg 1\right)$. For fractures of finite size, the rigid matrix prevents the fracture to shear whatever $k_{s}$, while for infinite fracture, the shear is possible on fracture planes with a displacement equal to the ratio $\tau / k_{s}$.

\subsection{Fracture Stiffness Length as an Indicator of the Partitioning Between Fracture Plane and Matrix} Elastic Resistance

Equation (13) emphasizes the partitioning in stress between elastic resistances of both the fracture surface stiffness on the one hand and the matrix deformation at fracture tips on the other hand, in a ratio equal to $\frac{k_{s}}{k_{m}}$, which depends on matrix and fracture properties, and on the fracture length $I_{f}$. To focus on the importance of fracture length, we define the stiffness length $I_{s}$ as the fracture length for which $k_{s}=k_{m}$ :

$$
I_{s}=\frac{E_{m}^{*}}{k_{s}}=\frac{3 \pi}{8} \cdot \frac{1-v_{m} / 2}{1-v_{m}^{2}} \frac{E_{m}}{k_{s}}
$$




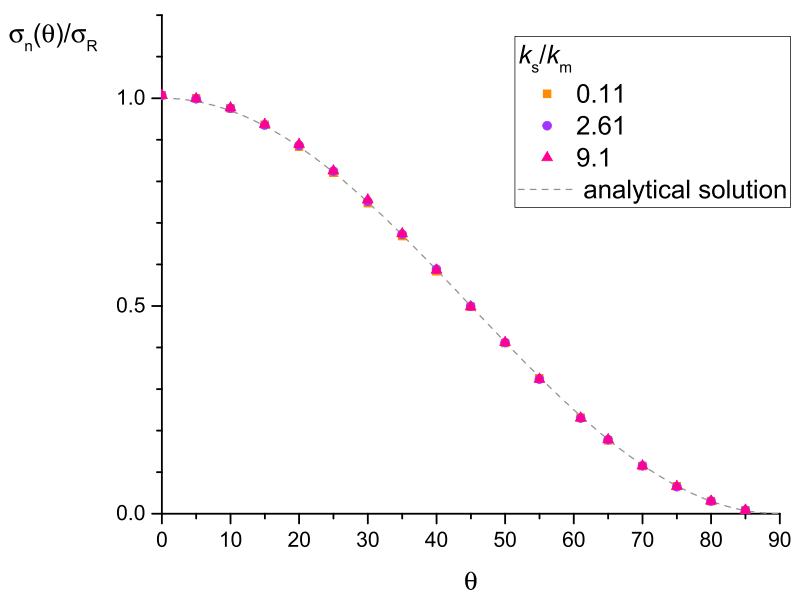

Figure 5. Normal stress versus fracture orientation for different values of the ratio $k_{s} / k_{m}$. The normal stress is normalized by the applied remote stress. The analytical solution is $\sigma_{n}(\theta)=\sigma_{r} \cos ^{2} \theta$.

$I_{S}$ is defined by intrinsic mechanical properties of both fracture and matrix.

For fractures much larger than $I_{s}$, most of the elastic resistance is due to the stiffness of the fracture surface (surface-dominated stiffness); for fractures much smaller than $I_{S}$, the resistance is due to the matrix deformation at fracture tips (matrix-dominated stiffness).

$I_{S}$ can be used to rewrite the stress-displacement relationship. For instance, for noncritically stressed fracture $\left(\tau_{f}<\tau_{p}\right)$, equation (13) writes as $\tau=\left(k_{s}+k_{m}\right) * \bar{t}$. Replacing $k_{s}$ by $I_{s}$ leads to

$$
\tau=E_{m}^{*}\left(\frac{1}{I}+\frac{1}{l_{s}}\right) * \bar{t}
$$

We will see in the next paragraphs that the concept of stiffness length is interesting to assess the contribution of the different elements of a fracture population to the bulk mechanical properties. Note that $k_{s}$ may depend on fracture length (Bandis et al., 1981; Barton, 1976; Fardin, 2003; Giwelli et al., 2014; Yoshinaka \& Yamabe, 1986; Yoshinaka et al., 1993), which would change the transition between matrix-dominated and surfacedominated fractures. But as long as $k_{s}$ does not decrease as $I^{-1}$, which seems the case, there exists a stiffness length $I_{S}$ that separates both the matrix- and stiffness-dominated regimes.

\subsection{The Stress Ellipsoid on the Fracture Plane}

Because of the matrix stiffness, the remote shear stress $\tau$ is different from the stress at the center of the fracture plane $\tau_{f}=k_{s} t_{f}$. For nonslipping fractures, $\tau_{f}$ can be deduced from $\tau$ by applying a coefficient $k_{s} /\left(k_{s}+k_{m}\right)$

$$
\tau_{f}=\tau \frac{k_{s}}{k_{s}+k_{m}}
$$

In contrast, the stress normal to the fracture plane is similar when measured on the center of the fracture disk or remotely (Figure 5).

The Mohr's circle derived from the shear and normal stress (Figure 6) calls for two remarks:

- The stress tensor at the fracture center is necessarily different from the remote stress. In particular, the deviatoric part is smaller.

- For different fracture orientations, the stress conditions at the fracture $\left(\tau_{f}, \sigma_{n}\right)$ center define an ellipse.

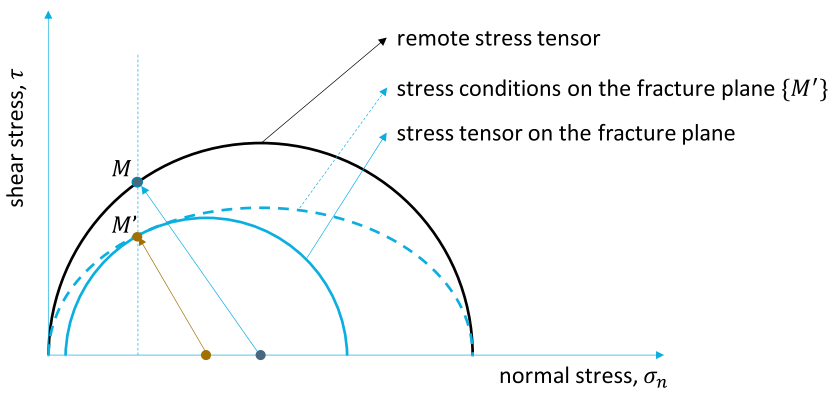

Figure 6. Illustration of the stress ellipse. For a given orientation (vertical dashed line), the remote stress tensor (solid black line) defines the remote shear and normal stress conditions (point $M$ ). The stress conditions on the fracture plane (point $M^{\prime}$ and blue solid line) are defined by the same normal stress as in $M$ but a shear stress that is reduced by a factor $\frac{k_{s}}{k_{s}+k_{m}}$ below the slip threshold. The blue dashed line indicates the stress conditions on the fracture plane for different orientations of fractures.

\subsection{The Mohr-Coulomb Envelope}

In the previous paragraphs, we introduced $\tau_{p}$ as the limit of the shear stress acting on the fracture plane, $\tau_{f}$, above which frictional slip is triggered. At the system boundary, this requires a stress larger than $\tau_{p}$, due to the stiffness of the matrix. According to equations (9) and (13), the critical stress for the boundary stress $\tau$ is $\tau_{p}^{*}$ such as

$$
\tau_{f}>\tau_{p} \Leftrightarrow \tau>\tau_{p}^{*}=\tau_{p} \frac{k_{s}+k_{m}}{k_{s}}
$$

If $k_{s} \gg k_{m}$, which also corresponds to very large fractures, the contribution of the matrix stress is negligible, and both thresholds are about similar $\tau_{p}^{*}=\tau_{p}$. The other end-member case with $k_{s} \ll k_{m}$ corresponds to free-slipping fracture, for which there is no reason to consider a threshold for slip $\left(\tau_{p}^{*} \rightarrow \infty\right)$. Figure 7 illustrates a case, where both contributions (fracture and matrix) are about similar. 


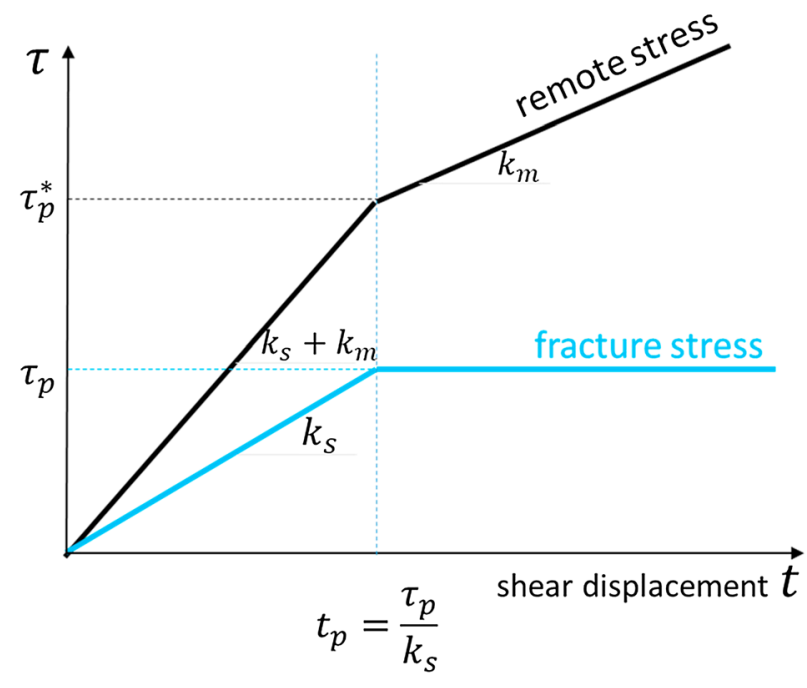

Figure 7. Shear stress as a function of displacement on the fracture plane (blue curve) and at the system boundary (black curve), which includes fracture and matrix deformation. The critical shear stress required to trigger fracture slip is $\tau_{p}^{*}$. It is larger than $\tau_{p}\left(\tau_{p}^{*}=\frac{k_{s}+k_{m}}{k_{s}} \tau_{p}\right)$ because of the matrix resistance. $t_{p}$ is the maximum shear displacement in the elastic regime.
Another consequence of matrix stresses is that the angle at which a fracture can slip must be predicted by the stress ellipse (see previous paragraph) rather than by the Mohr's circle. This is illustrated in Figure 8 top, where the dashed blue line indicates the orientation where the shear stress $\tau_{f}$ on the fracture plane reaches the slip threshold. It is different from the orientation that would be obtained by comparing the Mohr's circle and the Mohr-Coulomb slip envelope. The consequence in terms of fracture displacement is given in Figure 8 bottom.

\subsection{The Normal Displacement}

For the normal displacement, we obtain the same result except that both elastic components (stiffness of the fracture plane and matrix deformation) hardly operate together. If the normal stress $\sigma_{n}$ is positive, the fracture walls can hardly interpenetrate if the normal stiffness of the fracture walls $k_{n}$ is very large, and thus, the deformation in the surrounding matrix is limited. The displacement normal to the fracture plane $u_{f}$ is controlled by $k_{n}: u_{f}=\sigma_{n} / k_{n} . k_{n}$ is generally much larger than $k_{s}$ so that the displacement $u_{f}$ is small compared to the shear displacement $t_{f}$.

For tensional stress $\left(\sigma_{n}<0\right)$, the fracture walls separate and the total displacement is due to the resistance of the surrounding matrix $u_{f}=\sigma_{n}$ $/ k_{m}^{\prime}$, where $k_{m}^{\prime}$ is slightly different from $k_{m}$ (see equation (2))

$$
k_{m}^{\prime}=\frac{\pi}{4} \frac{1}{1-v_{m}^{2}} \frac{E_{m}}{I_{f}}
$$

\section{The Effective Elastic Properties of Rock Volume With a Population of Fractures}

The mechanical behavior of a rock mass depends on the elastic properties of the matrix and the contribution of individual fractures. We first develop the contribution of individual fractures to the total strain, and then we develop the theories that account for a large number of fractures. To simplify the equations, we calculate only the shear displacement on each fracture. The generalization to shear and normal displacements is, however, straightforward. Note that, for compressive systems with a wide range of orientations, the contribution of normal displacement to deformation is negligible compared to that of shear displacement.

\subsection{The Contribution of a Fracture to the Deformation of a Rock Volume}

We calculate the contribution to rock strain of a fracture, whose displacement at the fracture center is $t$ given any stress tensor $\overline{\bar{\sigma}}$ at the system boundary. For a fracture plane, whose normal vector is $\mathbf{n}$, the shear stress conditions are fully defined by the couple $\left(\tau, \sigma_{n}\right)$, whose expressions can be derived from the stress tensor $\overline{\bar{\sigma}}$ and $\mathbf{n}$

$$
\sigma_{n}=\mathbf{n}^{\top} \cdot \overline{\bar{\sigma}} \cdot \mathbf{n} \tau=\mathbf{n}^{\top} \cdot \overline{\bar{\sigma}} \cdot \mathbf{s}
$$

with the shear direction given by

$$
\mathbf{s}=\frac{\mathbf{s}_{\mathbf{g}}}{\left\|\mathbf{s}_{\mathbf{g}}\right\|} \mathbf{s}_{\mathbf{g}}^{\top}=\mathbf{n}^{\top} \cdot \overline{\bar{\sigma}} \cdot\left(\mathbf{1}-\mathbf{n} \otimes \mathbf{n}^{\top}\right)
$$

where 1 is the identity matrix.

The previous expressions are calculated in the fracture plane. The contribution of the fracture to the displacement of a specific boundary $X$ is obtained by projecting and integrating the displacement field on $X$ (see, e.g., Kachanov, 1980, 1992; Kachanov \& Sevostianov, 2013) 

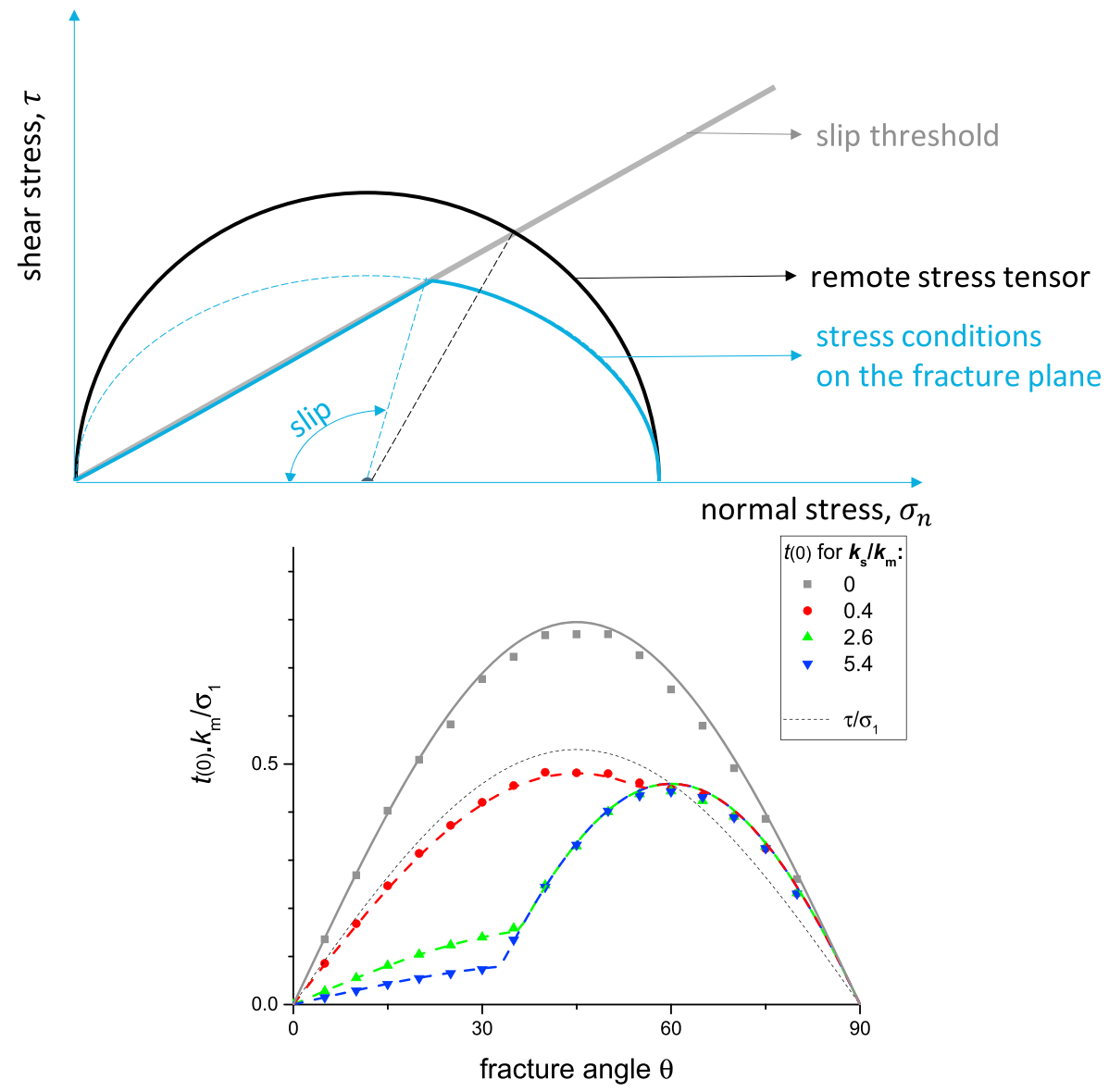

Figure 8. (top) Conditions of slip defined in a Mohr-Coulomb stress diagram (shear versus normal stress). The blue solid line defines the stress conditions on the fracture plane for different orientations. For angles where no slip occurs, it is defined by the minimum of the Mohr-Coulomb ellipse (Figure 6) and the slip threshold. The blue dashed line indicates the angle above which slip occurs. (bottom) Average displacement $t$ calculated at the fracture center for different angles to the main compressive stress axis $\sigma_{1}$. The displacement is normalized by $\sigma_{1} / k_{m}$. Fractures follow a linear Mohr-Coulomb slip criterion with a friction angle of $30^{\circ}$ and no cohesion. The four curves are plotted for different ratio $k_{s} / k_{m}$ as indicated in the framed box. The dots are calculated from 3DEC, while the lines are derived from equations (6) and (7). The break in the curves marks the angle limit between nonslipping and slipping conditions; the larger is $k_{s}$, the smaller the angle limit is. The black dashed line gives the ratio between the applied shear stress $\tau$ and $\sigma_{1}$.

$$
\mathbf{t}_{x}=\left(\mathbf{n} \cdot \mathbf{n}_{x}\right) \frac{\iint_{S} \mathbf{t} d S}{S_{x}}
$$

where $S_{X}$ and $\mathbf{n}_{x}$ are the surface and normal vector to $X$, respectively, $S$ is the plane that includes the fracture $F$, and $\mathbf{t}$ the displacement vector field in the plane. This expression is used to calculate the deformation of a sample, for which $X$ is a boundary and whose volume $V$ is $V=S_{X}{ }^{*} I_{X}$ with $I_{X}$ the dimension perpendicular to $X$ (i.e., in the direction $\mathbf{n}_{x}$ ). The contribution of the fracture to the deformation component $\epsilon_{x y}$, where $x$ refers to the surface $X$ and $y$ to a direction vector $\mathbf{n}_{\mathbf{y}}$, is

$$
\boldsymbol{\epsilon}_{x y}=\frac{\mathbf{t}_{x} \cdot \mathbf{n}_{y}}{I_{x}}=\left(\mathbf{n} \cdot \mathbf{n}_{x}\right) \frac{\iint_{S}\left(\mathbf{t} \cdot \mathbf{n}_{y}\right) \mathrm{d} S}{V}
$$

Since most of the displacement $\mathbf{t}$ that contributes to boundary displacements is within the fracture disk, the expression is generally expressed with the average displacement in the fracture disk $\overline{\mathbf{t}}_{f}$ and the fracture disk of area $S_{f}$ 
Table 1

List of Simulations Performed With the Itasca Software 3DEC $\odot$ to Calculate the Young's Modulus and Poisson's Ratio of a Series of Fractures Embedded in an Elastic Matrix

\begin{tabular}{|c|c|c|c|c|c|c|c|c|}
\hline Name & $\begin{array}{c}\text { Sample } \\
\text { dimensions }\left(\mathrm{m}^{3}\right)\end{array}$ & $\begin{array}{l}\text { Fracture } \\
\text { size }(m)\end{array}$ & $\begin{array}{l}\text { DFN density } \\
\left(p_{32} ; \mathrm{m}^{2} / \mathrm{m}^{3}\right)\end{array}$ & $\begin{array}{l}\text { DFN percolation } \\
\text { parameter }(-)\end{array}$ & $k_{\mathrm{s}}(\mathrm{GPa} / \mathrm{m})$ & $k_{m}(\mathrm{GPa} / \mathrm{m})$ & $\begin{array}{l}\text { Indicative number } \\
\text { of fractures (-) }\end{array}$ & $\begin{array}{c}\text { Number of } \\
\text { realizations (-) }\end{array}$ \\
\hline n01 & $8 \times 4 \times 4$ & 0.5 & 1 & 0.785 & $0 ; 12 ; 72$ & 117 & 890 & 10 \\
\hline $\mathrm{n} 02$ & $8 \times 4 \times 4$ & 1 & 1 & 1.571 & $0 ; 12 ; 72$ & 58 & 298 & 10 \\
\hline n03 & $8 \times 4 \times 4$ & 0.5 & 2 & 1.571 & $0 ; 12 ; 72$ & 117 & 1,700 & 10 \\
\hline n04 & $8 \times 4 \times 4$ & 0.5 & 3 & 2.356 & $0 ; 12 ; 72$ & 117 & 2,580 & 10 \\
\hline n06 & $8 \times 4 \times 4$ & 2 & 1 & 3.142 & $0 ; 12 ; 72$ & 29 & 97 & 10 \\
\hline n05 & $8 \times 4 \times 4$ & 1 & 2 & 3.142 & $0 ; 12 ; 72$ & 58 & 580 & 10 \\
\hline n08 & $8 \times 4 \times 4$ & 1 & 3 & 4.712 & $0 ; 12 ; 72$ & 58 & 835 & 10 \\
\hline n09 & $8 \times 4 \times 4$ & 2 & 2 & 6.283 & $0 ; 12 ; 72$ & 29 & 200 & 10 \\
\hline $\mathrm{n} 11$ & $8 \times 4 \times 4$ & 2 & 3 & 9.425 & $0 ; 12 ; 72$ & 29 & 320 & 10 \\
\hline $\mathrm{n} 12$ & $8 \times 4 \times 4$ & 2 & 5 & 15.708 & $0 ; 12 ; 72$ & 29 & 510 & 10 \\
\hline
\end{tabular}

Note. For all the simulations, the Young's modulus of the elastic matrix is $E_{m}=53 \mathrm{GPa}$, the Poisson's ratio $v_{m}=0.25$, and the normal stiffness $k_{n}=12,600 \mathrm{GPa} / \mathrm{m}$. DFN = discrete fracture network.

$$
\boldsymbol{\epsilon}_{x y}=\left(\mathbf{n} \cdot \mathbf{n}_{x}\right) \frac{S_{f}}{V}\left(\overline{\mathbf{t}}_{f} \cdot \mathbf{n}_{y}\right)
$$

For the sake of completeness, the stress components $\tau$ and $\sigma_{n}$ are defined by equation (18), the shear displacement of $\overline{\mathbf{t}_{f}}$ is parallel to the vector $\mathbf{s}$ defined in equation (19), and the normal component is parallel to the fracture normal $\mathbf{n}$.

\subsection{The Contribution of a Population of Fractures to the Deformation of a Rock Volume}

We calculate the effective elastic properties of a multifracture system by summing all the contributions of the different fractures to the displacement at the system boundary. We first estimate the Young's modulus and Poisson's ratio from analytical expressions derived from different theories, and we compare the predictions for a series of models given in Table 1 with calculations performed with 3DEC $\odot$ version 5.2 (Itasca Consulting Group, 2016; Figure 9). 3DEC is a three-dimensional numerical software based on the distinct element method for discontinuum modeling. 3DEC is based on a Lagrangian calculation scheme, which is well suited to model large movements and deformations, and an explicit solver.

Most of the effective theories are written by incrementally adding fractures in a medium, which is already damaged by others. The total deformation is the sum of two terms:

- The deformation of the damaged matrix, $(\epsilon)_{i}$, due to the remote stress tensor $\overline{\bar{\sigma}}$ applied on the equivalent medium constituted of $(i-1)$ fractures with equivalent elastic properties $(E)_{i}$ and $(v)_{i r}$

- plus the deformation induced by the displacement on the additional ith fracture.

Written in the same general way as equations (21) and (22), this gives

$$
\left(\epsilon_{x y}\right)_{i}=\left(\epsilon_{x y}\right)_{i-1}+\left(\mathbf{n}_{i} \cdot \mathbf{n}_{x}\right) \frac{S_{i}}{V}\left(\overline{\mathbf{t}}_{i} \cdot \mathbf{n}_{y}\right)
$$

$S_{i}$ is the surface of the fracture $i, \mathbf{n}_{i}$ its normal, and $\overline{\mathbf{t}}_{i}$ its displacement on the additional ith fracture.

The main assumptions of any effective theory (ET) aim at statistically evaluating the average displacement $\overline{\mathbf{t}}_{i}$ which depends on both the stress applied to the fracture and the properties of the medium that the fracture will deform. The former (stress) is controlled by the remote stress tensor $\overline{\bar{\sigma}}$ and to some extent by the fluctuations of stresses induced by the $(i-1)$ fractures. To our knowledge, the role of stress fluctuations is not usually considered in effective theories; it will not be either in this study. The latter (medium behavior) is controlled by the matrix properties $E_{m}$ and $v_{m}$, which may be altered by the previous $(i-1)$ fractures to some extent.

The shear component of $\overline{\mathbf{t}}_{i}$ can be evaluated from equation (12), which depends on three terms: the shear stress $\tau=\mathbf{n}_{i}^{\top} \cdot \overline{\bar{\sigma}} \cdot \mathbf{s}_{i}$ with $\mathbf{s}_{i}$ the direction of shear (equation (19)); the elastic shear stiffness $k_{s i}$ which may depend 

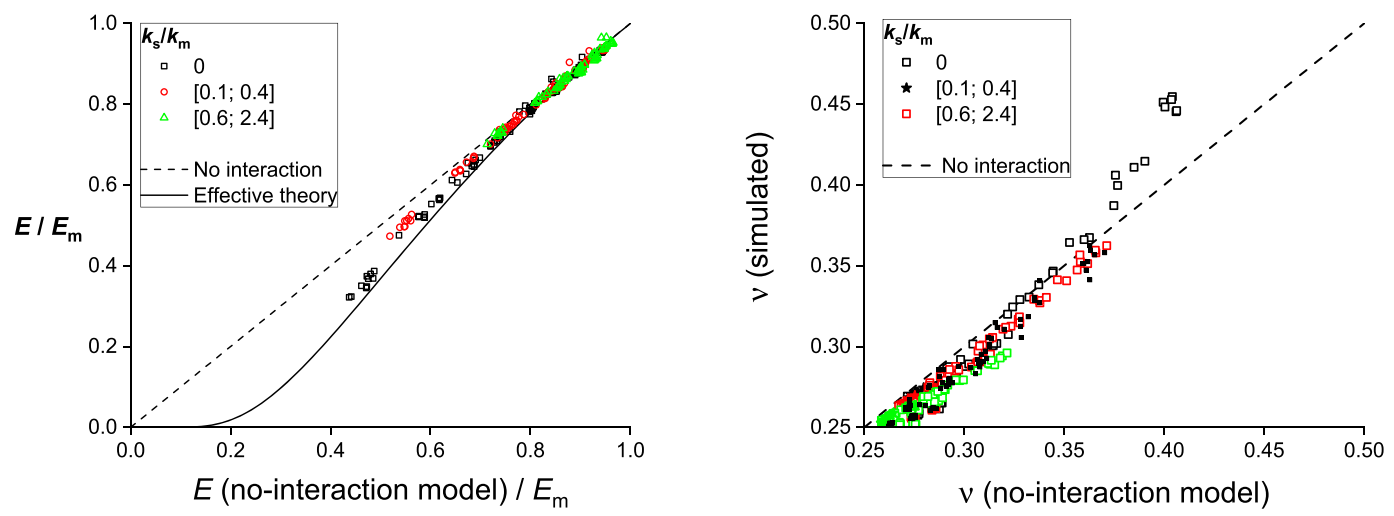

Figure 9. Young's modulus (left) and Poisson's ratio (right) calculated, in 3-D for all the cases shown in Table 1, as a function of the no-interaction model estimate. The dashed line shows the prediction by the no-interaction model. The full line on the left graph indicates the prediction by the effective theory for the case $k_{s}=0$.

on each fracture; and the local matrix-fracture stiffness $k_{m i}$ which is a function of the elastic properties of the medium surrounding the $i$ th fracture. With these assumptions, evaluating $\overline{\mathbf{t}}_{i}$ comes to assessing this latter term. The normal component of of $\overline{\mathbf{t}}_{i}$ is assumed to be equal to $\sigma_{n} / k_{n}$.

We write, as an example, the eventual deformation for noncritically stressed fractures, obtained by summing the contribution of all fractures:

$$
\left(\boldsymbol{\epsilon}_{x y}\right)_{i}=\left(\boldsymbol{\epsilon}_{x y}\right)_{m}+\sum_{i}\left(\mathbf{n}_{i} \cdot \mathbf{n}_{x}\right) \frac{S_{i}}{V}\left(\left(\frac{\mathbf{n}_{i}^{\top} \cdot \overline{\bar{\sigma}} \cdot \mathbf{s}_{i}}{k_{s i}+k_{m i}} \mathbf{s}_{i}+\frac{\mathbf{n}_{\mathbf{i}}^{\top} \cdot \overline{\bar{\sigma}} \cdot \mathbf{n}_{\mathbf{i}}}{k_{n i}} \mathbf{n}_{i}\right) \cdot \mathbf{n}_{y}\right)
$$

The elastic modulus and Poisson's ratio can be calculated by applying equation (24) to different boundary planes and directions. For a uniaxial compression $\sigma$ along the $x$ axis without confining pressure along $y$ and $z$ axes, the Young's modulus and Poisson's ratio can be calculated from

$$
(E)_{i}=\frac{\sigma_{x x}}{\epsilon_{x x}}(v)_{i}=\frac{-\epsilon_{z z}}{\epsilon_{x x}}
$$

With confining pressure, the apparent Young's modulus and Poisson's ratio are derived from the general equation $\epsilon_{i i}=\frac{1}{E}\left(\sigma_{i i}-v\left(\sigma_{j j}+\sigma_{k k}\right)\right)$, where $i, j$, and $k$ denote iteratively the $x, y$, and $z$ directions. In any cases, the total deformation is increased by the contribution of fracture displacements, so that the Young's modulus is smaller than $E_{m}$ and the Poisson's ratio is larger than $v_{m}$.
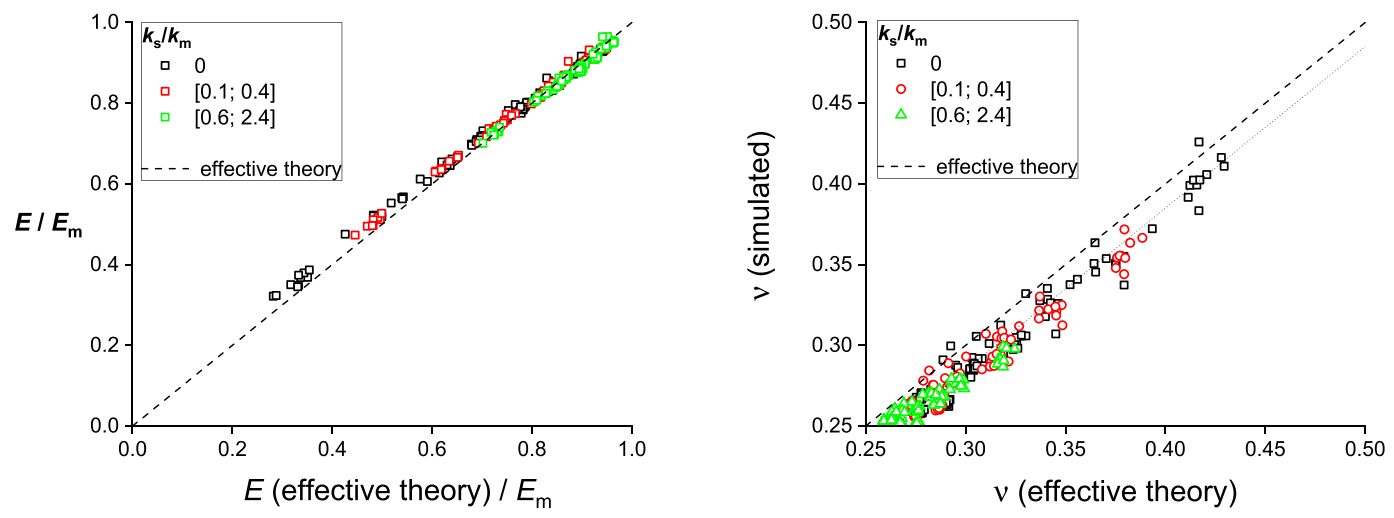

Figure 10. Young's modulus (left) and Poisson's ratio (right) calculated with 3DEC for all the cases shown in Table 1, as a function of the effective theory estimate. The dashed line shows the prediction $y=x$. The dotted line in the Poisson's ratio graph is $y=x-0.15$. 
We now review different theories that aim at predicting the Young's modulus and Poisson's ratio from equation (24). The differences stem from the way the matrix-fracture stiffness term $k_{m i}$ is calculated.

\subsubsection{No-Interaction Model}

The simplest model is to consider that the medium is not damaged at the vicinity of the fracture, so that the elastic properties are those of the intact matrix $E_{m}$ and $v_{m}$ (no-interaction model). This gives an expression of $k_{m i}$ similar to equation (3):

$$
k_{m i}=\frac{3 \pi}{8} \frac{1-\frac{v_{m}}{2}}{1-v_{m}^{2}} \frac{E_{m}}{l_{i}}
$$

where $l_{i}$ is the length of the $i$ th fracture.

The no-interaction model is a good description of systems with a small density of fractures, where any new fracture is on average surrounded by the intact elastic matrix. This also entails that the contribution of each fracture to $\left(\epsilon_{x y}\right)_{i}$ is independent of the other. It predicts well the Young's modulus of a fractured media when the density of fractures is small (i.e., fractures are far from each other). It gives a good estimate of the Poisson's ratio whatever the fracture density.

\subsubsection{The Effective Medium Theory}

The effective medium theories (see the review in Guéguen \& Kachanov, 2011; Jaeger et al., 2009; Kachanov, 1987 , for the case $k_{s}=0$ ) approximate the interaction between fractures with different schemes, the most popular of which being the self-consistent theory (O'Connell \& Budiansky, 1974) and the differential scheme (Hashin, 1988). Here we develop the differential scheme, which avoids some inconsistencies of the differential scheme at high crack densities (Bruner, 1976). It basically considers that the ith fracture is surrounded by a damaged medium with homogeneous properties, whose Young's modulus and Poisson's ratio are the average properties of the medium constituted by the $(i-1)$ fractures, $(E)_{i-1}$ and $(v)_{i-1}$, respectively:

$$
k_{m i}=\frac{3 \pi}{8} \frac{1-\frac{(v)_{i-1}}{2}}{1-(v)_{i-1}^{2}} \frac{(E)_{i-1}}{l_{i}}
$$

The effective medium theory predicts Young's modulus smaller than the no-interaction model since fractures are supposed to be embedded in a softer matrix entailing a larger displacement in fractures. Results from the ET with $k_{s}=0$ are reported in Figure 9 (solid line); they underpredict the Young's modulus whatever the fracture density, but the difference with simulations tends to be smaller and smaller when fracture density increases (i.e., when the Young's modulus decreases). Figure 10 shows two additional facts:

- The ET tends to the no-interaction model when $k_{s}$ increases, emphasizing the fact that the fracture plane resistances impede the interactions between fractures.

- If $k_{s}=0$, the results are independent of the fracture size distribution. The dependency on $k_{s}$ observed for $k_{s}>0$ is related to the fracture-size dependency of the ratio between $k_{s}$ and the matrix-fracture stiffness.

Note that all the results shown in this section have been obtained with a value of $k_{n} 175-1,000$ times larger than $k_{s}$ (see Table 1), precluding any significant contribution of the normal displacement compared to shear. 3.2.3. Shear Versus Normal Contribution to the Young's Modulus and Poisson's Ratio

The contribution of normal displacement to elastic parameters is likely varying with the ratio between $k_{n}$ and $k_{s}$. To assess it, we calculate the Young's modulus and Poisson's ratio in both ways, the first by taking both normal and shear components $\left(E\left(k_{s}, k_{n}\right)\right.$ and $v\left(k_{s}, k_{n}\right)$, respectively) and the second by taking only shear $\left(E\left(k_{s}\right)\right.$ and $v\left(k_{s}\right)$, respectively). The contribution of the normal displacement is then calculated as the difference between both expressions normalized by one of them: $C_{n}(E)=\left(E\left(k_{s}\right)-E\left(k_{s}, k_{n}\right)\right) / E\left(k_{s}\right)$ and $C_{n}(v)=\left(v\left(k_{s}\right)-v\left(k_{s}\right.\right.$, $\left.\left.k_{n}\right)\right) / v\left(k_{s}\right)$, for the Young's modulus and Poisson's ratio, respectively.

Figure 11 shows $C_{n}(E)$ and $C_{n}(v)$ calculated by using the effective-medium approximation as a function of the ratio $\kappa=k_{n} /\left(k_{s}+k_{m}\right)$ for different fracture densities and $k_{s}$ values. For both elastic parameters, the contribution varies as follows: $C_{n}(E)=1 / 3 \kappa$ and $C_{n}(v)=1 / 2 \kappa$.

A ratio of 100 between $k_{n}$ and $k_{s}$ gives a contribution of normal displacements of $0.3-0.5 \%$ for the largest fractures (for which $k_{s} \gg k_{m}$ ) and even less for the smaller ones. This justifies to neglect normal displacements in the deformation for most of the cases reported in the literature. 

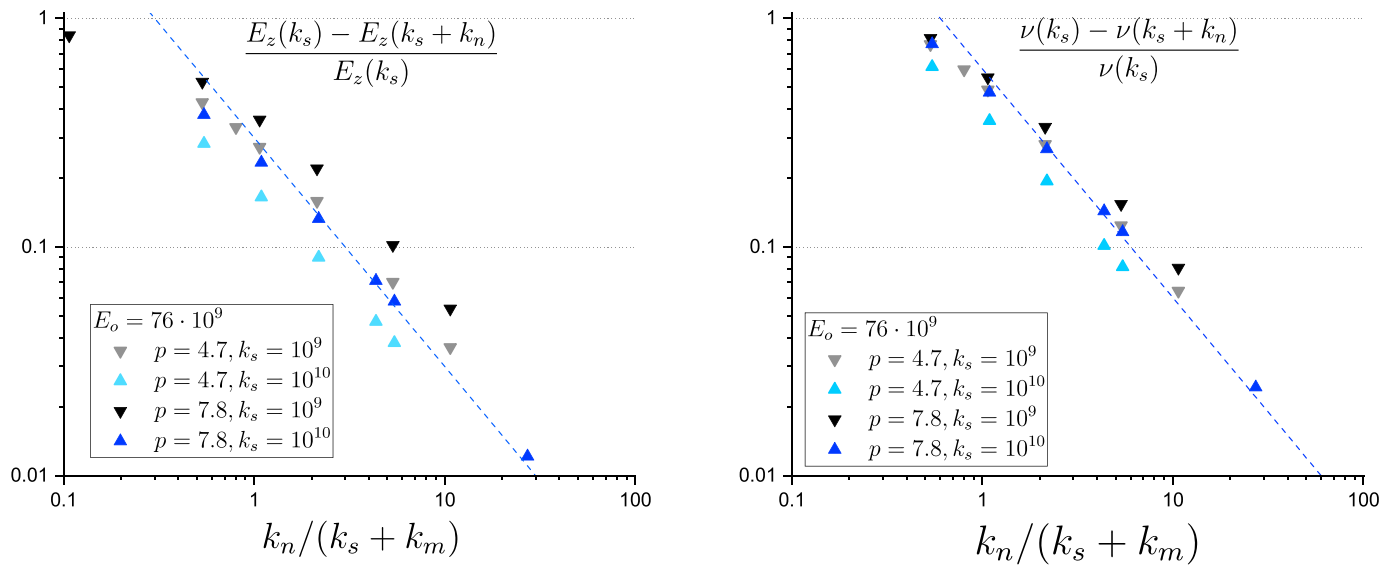

Figure 11. Contributions of the normal displacements to Young's modulus (left) and Poisson's ratio (right) as a function of the ration $k_{n} /\left(k_{s}+k_{m}\right)$. The way the normal contributions are calculated is given in the text. The simulations were performed by using the effective-medium approximation for two networks with a fracture size $I=1$ and percolation parameters of 4.7 and 7.8. For each network, the Young's modulus of the intact matrix is $76 \mathrm{GPa}$, and the Poisson's ratio is 0.25 . And two $k_{s}$ values were used of $10^{9}$ and $10^{10} \mathrm{GPa}$ (see the graph legends).

\section{Relationship Between Fracture Network Densities and Elastic Rock Mass Properties}

In this section, we link elastic rock mass properties with fracture network densities. We relate the results on the DFN description of fractured rock mass (Davy et al., 2013; Dershowitz \& Einstein, 1988; Elmo et al., 2014; Jing et al., 2007; Long et al., 1982; Painter \& Cvetkovic, 2005; Selroos et al., 2002), which is basically defined by a density distribution of fracture sizes and orientations $n(I, \theta)$ with $\theta$ the orientation vector that includes both the strike and dip.

Two DFN metrics are worth being mentioned (Maillot et al., 2016): the total fracture surface per unit volume (often named $p_{32}$ Dershowitz \& Herda, 1992), which controls permeability of dense networks (Kirkpatrick, 1973; Oda, 1985), and the percolation parameter $p$, which controls the network connectivity (Bour \& Davy, 1997, 1998; de Dreuzy et al., 2000; Nakaya \& Nakamura, 2007):

$$
p_{32}=\frac{\pi}{4 V} \int_{\theta} \int_{l} I^{2} n(I, \theta) \mathrm{d} / \mathrm{d} \theta \quad p=\frac{\pi^{2}}{8 \mathrm{~V}} \int_{\theta} \int_{l} I^{3} n(I, \theta) \mathrm{d} / \mathrm{d} \theta
$$

where $p$ is a dimensionless measure of the total volume occupied by fractures including overlaps; $p_{32}$ is the inverse of a length, which represents the average distance between fractures.

We first develop the case where no fracture is critically stressed from equation (24). We make a series of reasonable assumptions to develop analytical or semianalytical solutions:

1. We assume that fracture sizes and orientations are not correlated, so that density distribution can be written as $n(I, \theta)=n(I) \operatorname{pdf}(\theta)$, where $\operatorname{pdf}(\theta)$ is the probability density function of fracture orientations.

2. The stress-orientation term in equation (24) is $\mathrm{T}_{\theta}=\left(\mathbf{n}_{i}^{\top} \cdot \overline{\bar{\sigma}} \cdot \mathbf{s}(\theta)\right) \cdot\left(\mathbf{n}(\theta) \cdot \mathbf{n}_{x}\right)\left(\mathbf{s}(\theta) \cdot \mathbf{n}_{y}\right)$. We assume that $\mathrm{T}_{\theta}$ can be simplified to

$$
\mathrm{T}_{\theta}=\sigma s(\theta)
$$

where $\sigma$ is a value characterizing the applied stress.

3. The Young's modulus is calculated as

$$
E=\frac{\sigma}{\epsilon}
$$

where $\epsilon$ corresponds to the deformation in the adequate direction with respect to the stress tensor. We calculate the sum of fracture contributions to deformation by integrating equation (24) over the entire 
population of fractures. To account for a possible dependency of $k_{m}$ on the Young's modulus, as it is assumed in the ET, we rewrite equation (24) as a differential equation on the Young's modulus, where the incremental addition of a set of fractures leads to an incremental increase of the deformation $\epsilon$ and a consequent decrease of the Young's modulus:

$$
\mathrm{d}^{2} \epsilon=\sigma \cdot \mathrm{d}\left(\frac{1}{E}\right)=\sigma(s(\theta) \operatorname{pdf}(\theta) \mathrm{d} \theta) \frac{\pi}{4 V} \frac{I^{2} n(I) \mathrm{d} l}{k_{s}+k_{m}(I)}
$$

The integral over the $\theta$ term can be done independently, which leads to a size-dependent differential equation:

$$
\mathrm{d}\left(\frac{1}{E}\right)=F_{\theta} \cdot \frac{\pi}{4 V} \frac{I^{2} n(I) \mathrm{d} l}{\left(k_{s}+k_{m}(I)\right)}
$$

$F_{\theta}=\int_{\theta} s(\theta) \operatorname{pdf}(\theta) \mathrm{d} \theta$ is an orientation factor that takes into account both fracture and stress orientations. For uniaxial compression and uniformly distributed orientations, $F_{\theta}=\frac{2}{15}$. Integrating equation (30) is straightforward for both end-member cases, where $k_{s}$ is much larger or much smaller than $k_{m}$.

\subsection{Case of $\boldsymbol{k}_{\boldsymbol{m}} \ll \boldsymbol{k}_{\mathrm{s}}$}

If $k_{m}(I) \ll k_{s} \forall I$, fracture stiffness controls the total stress resistance; the integral of equation (30) leads to:

$$
\frac{1}{E}=\frac{1}{E_{m}}+\frac{F_{\theta} p_{32}}{k_{s}}
$$

The integral does not require any assumption on the matrix elastic properties, so it is valid for both the nointeraction model and the ET.

\subsection{Case of $\boldsymbol{k}_{m} \gg \boldsymbol{k}_{s}$}

This case $k_{m}(I) \gg k_{s} \forall /$ corresponds to frictionless cracks and have already been dealt with extensively in many studies, either from energy considerations (Budiansky \& O'Connell, 1976; Jaeger et al., 2009; O'Connell \& Budiansky, 1974; Walsh, 1965c) or by following the same approach as described here based on the Green's function of Fabrikant (1988; Guéguen \& Kachanov, 2011; Kachanov, 1992, 1993; Sayers \& Kachanov, 1995; Schoenberg \& Sayers, 1995).

In this case, matrix deformation controls the total stress resistance; equation (30) can be written as follows:

$$
\mathrm{d}\left(\frac{1}{E}\right)=F_{\theta} \cdot \frac{\pi}{4 V} \frac{I^{3} n(I) \mathrm{d} l}{E_{m}^{*}}
$$

With the no-interaction hypothesis, $E_{m}^{*}\left(=k_{m} * l\right.$; see equation (4)) is constant, and the integral gives

$$
\frac{1}{E}=\frac{1}{E_{m}}+\frac{2}{\pi} F_{\theta} \frac{p}{E_{m}^{*}}
$$

In the ET framework, $E_{m}^{*}$ reflects the properties of the damaged matrix $E$ and $v$. We rewrite equation (30) as follows:

$$
\mathrm{d}\left(\frac{1}{E}\right)=F_{\theta} \cdot \frac{\pi}{4 V} \frac{I^{3} n(I) \mathrm{d} I}{N(v) E} N(v)=\frac{3 \pi}{8}\left(\frac{1-v / 2}{1-v^{2}}\right)
$$

$N(v)$ is varying between 1.099 and 1.178 when $v$ varies between 0.0 and 0.5 , so it is not a big assumption to assume it constant and equal to its average value $\langle N(v)\rangle=N_{a} \sim 1.125$. Then we can rewrite the differential equation (32) 


$$
E \cdot \mathrm{d}\left(\frac{1}{E}\right)=-\mathrm{d} \ln E=\frac{F_{\theta}}{N_{a}} \cdot \frac{\pi}{4 V} P^{3} n(I) \mathrm{d} l
$$

whose integral expression is

$$
E=E_{m} \exp \left(-\frac{2 F_{\theta}}{\pi N_{a}} \cdot p\right)
$$

For uniformly distributed orientations, the constant term in the exponential function is $\frac{2 F_{\theta}}{\pi N_{a}} \cong 0.0754$. Expressions (33) and (36) are consistent with the references cited in the first paragraph of this section. Note that $F_{\theta} p$ is an extension of the percolation parameter that takes into account the orientations of fractures with respect to stress and boundaries.

\subsection{General Case}

In the general case, equation (30) can be solved semianalytically with either the no-interaction hypothesis or the assumptions of the ET. The Young's modulus results from the contribution of small fractures, whose deformation is dominated by the deformation of the surrounding matrix, and large ones, whose deformation is due to the stiffness and the friction on the fracture walls. The limit between both groups is the stiffness length $I_{S}$ defined in equation (14). For the no-interaction model, an approximate solution can be used to either equation (31) or (33) for the group of fractures larger, or smaller, than $I_{s}$. This gives

$$
\frac{1}{E}=\frac{1}{E_{m}}+F_{\theta}\left(\frac{p_{32}\left(I>I_{s}\right)}{k_{s}}+\frac{2}{\pi} \frac{p\left(I<I_{s}\right)}{E_{m}^{*}}\right)
$$

where $p_{32}\left(I>I_{S}\right)$ is the surface of all fractures larger than $I_{S}$ divided by the total volume; $p\left(I<I_{S}\right)$ is the percolation parameter for fracture smaller than $I_{s}$. In most of the cases, the prediction gives a fairly good approximation of the actual integral. This point will be developed in the next paragraph for power law length distribution.

We also derive an approximate analytical solution for the ET by calculating first the deformation due to fractures larger than $I_{S}$ from equation (33) and then by considering this value as the initial elastic property of the damaged medium for smaller fractures in equation (31). The eventual result gives

$$
E=\frac{E_{m} \exp \left(-\frac{2 F_{\theta}}{\pi N_{a}} p\left(I<I_{S}\right)\right)}{1+\frac{F_{\theta} p_{32}\left(I>I_{S}\right) E_{m}}{k_{s}}}
$$

Introducing small fractures first and then large ones would give

$$
E=\frac{E_{m} \exp \left(-\frac{2 F_{\theta}}{\pi N_{a}} p\left(I<I_{S}\right)\right)}{1+\frac{F_{\theta} p_{32}\left(I>I_{S}\right) E_{m}}{k_{S}} \exp \left(-\frac{2 F_{\theta}}{\pi N_{a}} p\left(I<I_{S}\right)\right)}
$$

The difference between both expressions is the exponential term in the denominator of equation (39). The expression obtained by introducing small fractures first (equation (39)) predicts a slightly larger value than equation (40). For the density distributions studied in the next paragraph, the difference between both expressions is less than $10 \%$.

\section{Application to Geologically Relevant Fracture Distribution}

Geological fractures are complex, ubiquitous, and observables at all scales (Tchalenko, 1970). This is one reason to consider power laws as good candidates for the distributions of fracture sizes (Bonnet et al., 2001). Another argument is that power law distributions emerge from the analysis of fracture maps at different scales (Bonnet et al., 2001; Bour et al., 2002; Darcel et al., 2006; Fox et al., 2007; Odling, 1997), as well as from mechanistic models of fracture growth (Davy et al., 2010, 2013). Power laws are the only distributions that 
have no characteristic scale, except their upper and lower bounds, which poses the issue of the scales that control fracture network properties.

A special case is obtained when the exponent of the power law size distribution lies in the range -3 to -4 . For this range, the density parameter $p_{32}$, which represents the total fracture surface and the second moment of the size distribution, is dominated by the lower bound of the size distribution; the percolation parameter, which represents the total volume surrounding fractures and the third moment of the size distribution, is dominated by the upper bound. The consequence is that the Young's modulus of fracture networks with this size distribution is entirely controlled by $I_{S}$. This can be shown by developing equation (37), which is an approximate solution for the no-interaction model, for $n(I)=\alpha V I^{-a}$ and $\left.a \in\right] 3,4[$.

$$
\frac{1}{E}=\frac{1}{E_{m}}+F_{\theta}\left(\frac{p_{32}\left(I>I_{s}\right)}{k_{s}}+\frac{2}{\pi} \frac{p\left(I<I_{s}\right)}{E_{m}^{*}}\right) \cong \frac{1}{E_{m}}+\frac{\pi}{4} \alpha F_{\theta} I_{s}^{-a+3}\left(\frac{1}{(a-3) k_{s}}+\frac{I_{s}}{(4-a) E_{m}^{*}}\right)
$$

The expression has been obtained by neglecting the smallest term of each integral $p_{32}$ and $p$. In the special cases where $a=3$ or $a=4$, the bounds of the integral cannot be neglected anymore. For both cases, the full equation (30) can be integrated for the no-interaction model, which gives:

for $a=3$,

$$
\frac{1}{E}=\frac{1}{E_{m}}+\frac{\pi}{4} \frac{\alpha F_{\theta}}{k_{s}} \log \left(\frac{I_{s}+I_{\text {max }}}{I_{s}+I_{\min }}\right)
$$

for $a=4$

$$
\frac{1}{E}=\frac{1}{E_{m}}+\frac{\pi \alpha F_{\theta}}{4} \frac{1+I_{S} / I_{\min }}{E_{m}^{*}} \log \left(\frac{1+I_{S} / I_{\max }}{1+s}\right)
$$

$I_{\max }$ and $I_{\min }$ are the largest and smallest fracture sizes, respectively. If $I_{\max }$ is much larger than $I_{M}$ and $I_{\min }$ much smaller, we obtain simplified relationships as follows:

for $a=3$,

$$
\frac{1}{E} \sim \frac{1}{E_{m}}+\frac{\pi}{4} \frac{\alpha F_{\theta}}{k_{s}} \log \left(\frac{I_{\max }}{I_{s}}\right)
$$

for $a=4$

$$
\frac{1}{E} \sim \frac{1}{E_{m}}+\frac{\pi}{4} \frac{\alpha F_{\theta}}{E_{m}^{*}} \log \left(\frac{I_{s}}{I_{\min }}\right)
$$

For $a=3$, large fractures are dominant, and the Young's modulus depends on the fracture stiffness $k_{s}$ and (logarithmically) on all the scales between $I_{S}$ and the largest fracture $I_{\max }$. For $a=4$, the Young's modulus depends on the matrix properties $E_{m}^{*}$ and (logarithmically) on all the scales between $I_{\min }$ and $I_{s}$. The dependency on small (large, respectively) fractures is even higher if the exponent $a$ is larger than 4 (respectively smaller than 3 ).

All these results are qualitatively similar for the ET. As an illustration of the above analysis, we show in Figure 12 the Young's modulus calculated with the ET from equations (24) and (27) for different exponents of the power law fracture size distribution. The Young's modulus is plotted as a function of $p_{32}$ (left), of the percolation parameter $p$ (middle), and of the parameter $p_{k}$ that derives from equation (37):

$$
p_{k}=\frac{p_{32}\left(I>I_{S}\right)}{k_{s}}+\frac{2 p\left(I<I_{S}\right)}{\pi}
$$

From the three parameters, $p_{k}$ is by far the best parameter to describe univocally the Young's modulus evolution for different fracture size distribution and mechanical properties (i.e., different values of $k_{s}$ ). A good fit of the so calculated Young's modulus is obtained by the following equation (dashed line, Figure 12 right):

$$
\frac{1}{E}=\frac{1}{E_{m}}+0.8 * F_{\theta} * p_{k}
$$

The preceding discussion emphasizes the critical contribution of fractures whose length is close to $I_{S}$ in the elastic properties of rock masses. In hardrock geological systems (gneissic or granitic), we estimate $I_{S}$ to 

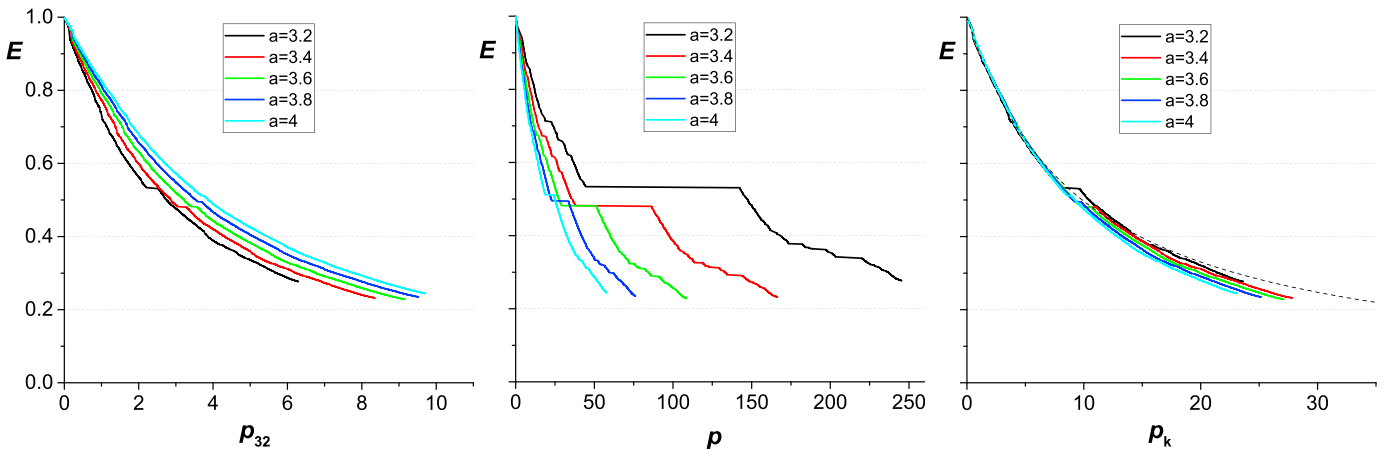

Figure 12. Plot of the Young's modulus $E$ calculated from the effective theory for fracture networks with power law size distributions. The stress conditions are similar to those described in the previous figures. $E$ is plotted versus $p_{32}$ (left), the percolation parameter $p$ (middle), and $p_{k}$ defined in equation (43); right). The dashed line in the graph to the right is given by equation (41).

range between 1 and $50 \mathrm{~m}$, taking reasonable values of the matrix Young's modulus ( $30-80 \mathrm{GPa}$ ) and of $k_{s}(3-$ $30 \mathrm{GPa} / \mathrm{m}$; Grasselli \& Egger, 2003; Yoshinaka \& Yamabe, 1986). A more complete discussion on $I_{s}$ will be given in a further study.

This is a preliminary attempt to apply the model to geological cases. Further discussions will be pursued in subsequent studies from field example cases that take into account measured fracture-size distribution and mechanical parameters (including the dependency of $k_{s}$ on fracture size).

\section{Conclusion}

The objective of this paper was to derive the relationships that link the elastic properties of rock masses to the geometrical properties of fracture networks, with a special emphasis to the case of frictional crack surfaces that is relevant to geological applications. For simplicity, we consider fracture networks to be made of disk-shaped cracks. We extend the well-known elastic solutions for free-slipping cracks to fractures whose plane resistance is defined by an elastic fracture (shear) stiffness $k_{s}$ and a stick-slip Coulomb threshold. Together with the elastic matrix Young's modulus and Poisson's ratio, $k_{s}$ defines a characteristic fracture size $I_{S}$ (called the stiffness length), below which the rock mass elastic behavior is dominated by the rock matrix deformation and above which is controlled by the resistance on the fracture plane.

A complete set of analytical solutions have been derived for the shear displacement in the fracture plane for stresses below the slip threshold and above, including the variations of displacement in the fracture plane and the relationship between stress and average displacement. All the expressions have been checked with numerical simulations. From these, we derive a simple expression of the stress partitioning between the resistances of the fracture plane on the one hand and of the elastic matrix on the other hand. We demonstrate that the stress conditions on the fracture plane define a stress ellipse, which derives from the remote Mohr's circle. The remote conditions for triggering slip must take into account not only the resistance of the fracture plane but also the mechanical resistance of the surrounding matrix. This has both consequences: (i) the remote stress threshold is larger than the fracture plane stress threshold by a ratio $\frac{k_{s}+k_{m}}{k_{s}}$, where $k_{m}$ is the matrix-fracture stiffness (i.e., the ratio between stress and displacement for free-slipping fractures) and (ii) the angle at which fracture can slip must be predicted from the stress ellipse rather than from the remote stress Mohr's circle.

The Young's modulus and Poisson's ratio were also derived for a rock mass with a population of fractures, with the intrinsic difficulty to describe properly the fracture interactions. In the case of large $k_{s}$ values $\left(k_{s} \gg k_{m}\right)$, the bulk elastic modulus is controlled by the total fracture surface and more precisely by the ratio $p_{32} / k_{5}$, where $p_{32}$ is the total fracture surface divided by the sample volume; this is the case of fractures larger than the stiffness length $I_{s}$. This result differs from the slipping case $\left(k_{s} \ll k_{m}\right)$, where the elastic moduli is controlled by the percolation parameter, that is, by the third moment of the fracture size distribution; this is the case for fractures smaller than $I_{s}$. For a complete fracture size distribution, the elastic modulus can be efficiently 
deduced from a combination of these density parameters, provided that they are calculated for the right subset of the fracture size distribution: $p_{32} / k_{s}$ for large fractures $\left(>I_{S}\right)$ and $p$ for small fractures $\left(<I_{S}\right)$.

Thanks to a comparison with numerical simulations, we show that the ET gives a very good approximation of the bulk elastic properties. ET can be calculated analytically by introducing fractures one by one and assuming that the surrounding elastic matrix has the property of the bulk damaged medium.

These results were applied to power law fracture size distributions, which are likely relevant to geological cases. We show that if the power law fracture size exponent is in the range -3 to -4 , which corresponds to a wide range of geological fracture networks, the elastic properties of the bulk rock are almost exclusively controlled by $k_{s}$ and $I_{s}$, meaning that the fractures of size $I_{s}$ play a major role in the definition of the elastic properties. In hardrock geological systems (gneissic or granitic), we estimate $I_{S}$ to range between 1 and $50 \mathrm{~m}$.

These results are obtained for constant $k_{s}$, but a dependency of $k_{s}$ with fracture size or normal stress can be implemented straightforwardly, and it does not change the general features, that is, the control of elastic properties by a stiffness length —as long as the dependency of $k_{s}$ with fracture size does not call into question the existence of $I_{S}$.

\section{Acknowledgments}

This work was partially supported by the Swedish Nuclear Fuel and Waste Management Co. (SKB) and Posiva Oy. The authors are grateful to five anonymous and the Associate Editor for their comments that help improve the manuscript. We also like to thank Johannes Suikkanen for constructive discussions. The files that contain results from simulations and theories can be downloaded at https://mycore.corecloud.net/index.php/s/ hiPIAE06dIKS3Nh.

\section{References}

Amadei, B., \& Goodman, R. (1981). A 3-D constitutive relation for fractured rock masses. Studies in Applied Mechanics, Part B, 5, $249-268$. Atkinson, B. K. (1987). Fracture mechanics of rock (p. ii). London: Academic Press. https://doi.org/10.1016/B978-0-12-066266-1.50001-6

Audoly, B. (2000). Asymptotic study of the interfacial crack with friction. Journal of the Mechanics and Physics of Solids, 48(9), 1851-1864. https://doi.org/10.1016/S0022-5096(99)00098-8

Bandis, S., Lumsden, A. C., \& Barton, N. R. (1981). Experimental studies of scale effects on the shear behaviour of rock joints. International Journal of Rock Mechanics and Mining Sciences, 18(1), 1-21. https://doi.org/10.1016/0148-9062(81)90262-X

Bandis, S. C., Lumsden, A. C., \& Barton, N. R. (1983). Fundamentals of rock joint deformation. International Journal of Rock Mechanics and Mining Sciences, 20(6), 249-268. https://doi.org/10.1016/0148-9062(83)90595-8

Barber, J. R. (2003). Bounds on the electrical resistance between contacting elastic rough bodies. Proceedings of the Royal Society of London, Series A: Mathematical, Physical and Engineering Sciences, 459(2029), 53-66. https://doi.org/10.1098/rspa.2002.1038

Barton, N. (1976). The shear strength of rock and rock joints. International Journal of Rock Mechanics and Mining Sciences, 13(9), 255-279. https://doi.org/10.1016/0148-9062(76)90003-6

Barton, N., Lien, R., \& Lunde, J. (1974). Engineering classification of rock masses for the design of tunnel support. Rock Mechanics, 6(4), 189-236. https://doi.org/10.1007/BF01239496

Bieniawski, Z. (1973). Engineering classification of jointed rock masses. Civil Engineer in South Africa, 15(12), 335-344.

Bieniawski, Z. (1978). Determining rock mass deformability: Experience from case histories. Paper presented at Int. J. Rock Mech. Min. Sci., Elsevier.

Bonnet, E., Bour, O., Odling, N., Davy, P., Main, I., Cowie, P., \& Berkowitz, B. (2001). Scaling of fracture systems in geological media. Reviews of Geophysics, 39(3), 347-383. https://doi.org/10.1029/1999RG000074

Bour, O., \& Davy, P. (1997). Connectivity of random fault networks following a power law fault length distribution. Water Resources Research, 33(7), 1567-1583. https://doi.org/10.1029/96WR00433

Bour, O., \& Davy, P. (1998). On the connectivity of three-dimensional fault networks. Water Resources Research, 34(10), 2611-2622. https://doi. org/10.1029/98WR01861

Bour, O., Davy, P., Darcel, C., \& Odling, N. (2002). A statistical scaling model for fracture network geometry, with validation on a multiscale mapping of a joint network (Hornelen Basin, Norway). Journal of Geophysical Research, 107(B6), 2113. https://doi.org/10.1029/2001JB000176

Bruner, W. M. (1976). Comment on 'seismic velocities in dry and saturated cracked solids' by Richard J. O'Connell and Bernard Budiansky. Journal of Geophysical Research, 81(14), 2573-2576. https://doi.org/10.1029/JB081i014p02573

Budiansky, B., \& O'Connell, R. J. (1976). Elastic moduli of a cracked solid. International Journal of Solids and Structures, 12(2), 81-97. https://doi. org/10.1016/0020-7683(76)90044-5

Byerlee, J. D., \& Brace, W. F. (1968). Stick slip, stable sliding, and earthquakes_Effect of rock type, pressure, strain rate, and stiffness. Journal of Geophysical Research, 73(18), 6031-6037. https://doi.org/10.1029/JB073i018p06031

Comninou, M. (1977). Interface crack with friction in the contact zone. Journal of Applied Mechanics, 44(4), 780-781. https://doi.org/10.1115/ 1.3424179

Comninou, M., \& Dundurs, J. (1980). Effect of friction on the interface crack loaded in shear. Journal of Elasticity, 10(2), 203-212. https://doi. org/10.1007/bf00044504

Darcel, C., Davy, P., Bour, O., \& De Dreuzy, J. (2006). Discrete fracture network for the Forsmark site Rep. R-06-79 (p. 94). Stockhölm: Svensk Kärnbränslehantering $A B$.

Davy, P., Le Goc, R., \& Darcel, C. (2013). A model of fracture nucleation, growth and arrest, and consequences for fracture density and scaling Journal of Geophysical Research: Solid Earth, 118, 1393-1407. https://doi.org/10.1002/jgrb.50120

Davy, P., le Goc, R., Darcel, C., Bour, O., de Dreuzy, J.-R., \& Munier, R. (2010). A likely universal model of fracture scaling and its consequence for crustal hydromechanics. Journal of Geophysical Research, 115, B10411. https://doi.org/10.1029/2009JB007043

de Dreuzy, J. R., Davy, P., \& Bour, O. (2000). Percolation parameter and percolation-threshold estimates for three-dimensional random ellipses with widely scattered distributions of eccentricity and size. Physical Review E, 62(5), 5948-5952. https://doi.org/10.1103/ PhysRevE.62.5948

Dershowitz, W., \& Einstein, H. (1988). Characterizing rock joint geometry with joint system models. Rock Mechanics and Rock Engineering, 21(1), 21-51. https://doi.org/10.1007/BF01019674

Dershowitz, W. S., \& Herda, H. H. (1992). Interpretation of fracture spacing and intensity. Paper presented at Rock Mechanics, Balkema, Rotterdam. 
Elmo, D., Rogers, S., Stead, D., \& Eberhardt, E. (2014). Discrete fracture network approach to characterise rock mass fragmentation and implications for geomechanical upscaling. Mining Technology, 123(3), 149-161. https://doi.org/10.1179/1743286314y.0000000064

Fabrikant, V. (1990). Complete solutions to some mixed boundary value problems in elasticity. Advances in Applied Mechanics, 27, $153-223$.

Fabrikant, V. I. (1988). Green's functions for a penny-shaped crack under normal loading. Engineering Fracture Mechanics, 30(1), 87-104. https://doi.org/10.1016/0013-7944(88)90257-3

Fardin, N. (2003). The effect of scale on the morphology, mechanics and transmissivity of single rock fractures (PhD thesis, $157 \mathrm{pp}$.$) . Stockholm:$ Sweden KTH Royal Institute of Technology.

Fox, A., La Pointe, P., Hermanson, J., \& Öhman, J. (2007). Statistical geological discrete fracture network model. Forsmark modelling stage 2.2Rep (pp. 1-271). Stockholm, Sweden: Svensk Kärnbränslehantering AB.

Gambarotta, L., \& Lagomarsino, S. (1993). A microcrack damage model for brittle materials. International Journal of Solids and Structures, 30(2), 177-198. https://doi.org/10.1016/0020-7683(93)90059-G

Giwelli, A. A., Sakaguchi, K., Gumati, A., \& Matsuki, K. (2014). Shear behaviour of fractured rock as a function of size and shear displacement. Geomechanics and Geoengineering, 9, 253-264. https://doi.org/10.1080/17486025.2014.884728

Glubokovskikh, S., Gurevich, B., Lebedev, M., Mikhaltsevitch, V., \& Tan, S. (2016). Effect of asperities on stress dependency of elastic properties of cracked rocks. International Journal of Engineering Science, 98, 116-125. https://doi.org/10.1016/j.ijengsci.2015.09.001

Goodman, R. E., Taylor, R. L., \& Brekke, T. L. (1968). A model for the mechanics of jointed rocks. Journal of Soil Mechanics \& Foundations Division, 94(3), 637-660.

Grasselli, G., \& Egger, P. (2003). Constitutive law for the shear strength of rock joints based on three-dimensional surface parameters. International Journal of Rock Mechanics and Mining Sciences, 40(1), 25-40. https://doi.org/10.1016/S1365-1609(02)00101-6

Grechka, V., \& Kachanov, M. (2006). Effective elasticity of fractured rocks: A snapshot of the work in progress. Geophysics, 71(6), W45-W58. https://doi.org/10.1190/1.2360212

Griffith, W. A., Sanz, P. F., \& Pollard, D. D. (2009). Influence of outcrop scale fractures on the effective stiffness of fault damage zone rocks. Pure and Applied Geophysics, 166(10-11), 1595-1627. https://doi.org/10.1007/s00024-009-0519-9

Guéguen, Y., \& Kachanov, M. (2011). Effective elastic properties of cracked rocks-An overview. In Y. Leroy \& F. Lehner (Eds.), Mechanics of crustal rocks (pp. 73-125). Vienna: Springer. https://doi.org/10.1007/978-3-7091-0939-7_3

Halm, D., \& Dragon, A. (1998). An anisotropic model of damage and frictional sliding for brittle materials. European Journal of Mechanics A/Solids, 17(3), 439-460. https://doi.org/10.1016/S0997-7538(98)80054-5

Hashin, Z. (1988). The differential scheme and its application to cracked materials. Journal of the Mechanics and Physics of Solids, 36(6), 719-734. https://doi.org/10.1016/0022-5096(88)90005-1

Hoek, E. (1994). Strength of rock and rock masses. ISRM News Journal, 2(2), 4-16.

Hoek, E., \& Diederichs, M. S. (2006). Empirical estimation of rock mass modulus. International Journal of Rock Mechanics and Mining Sciences, 43(2), 203-215. https://doi.org/10.1016/j.jirmms.2005.06.005

Hoek, E., Kaiser, P. K., \& Bawden, W. F. (1995). Support of underground excavations in hard rock.

Horii, H., \& Nemat-Nasser, S. (1983). Overall moduli of solids with microcracks: Load-induced anisotropy. Journal of the Mechanics and Physics of Solids, 31(2), 155-171. https://doi.org/10.1016/0022-5096(83)90048-0

Israelsson, J. I. (1996). Short descriptions of UDEC and 3DEC. Developments in Geotechnical Engineering, 79, 523-528. https://doi.org/10.1016/ S0165-1250(96)80041-1

Itasca Consulting Group (2016). Three dimensional distinct element code, version 5.2. Minneapolis, MN.

Jaeger, J. C., Cook, N. G., \& Zimmerman, R. (2009). Fundamentals of rock mechanics. John Wiley \& Sons.

Jing, L., Stephansson, O., Lanru, J., \& Ove, S. (2007). Discrete fracture network (DFN) method. In Developments in geotechnical engineering (pp. 365-398). Amsterdam: Elsevier.

Kachanov, M. (1980). Continuum model of medium with cracks. Journal of the Engineering Mechanics Division, 106(5), $1039-1051$.

Kachanov, M. (1987). Elastic solids with many cracks: A simple method of analysis. International Journal of Solids and Structures, 23(1), 23-43. https://doi.org/10.1016/0020-7683(87)90030-8

Kachanov, M. (1992). Effective elastic properties of cracked solids: Critical review of some basic concepts. Applied Mechanics Reviews, 45(8), 304-335. https://doi.org/10.1115/1.3119761

Kachanov, M. (1993). Elastic solids with many cracks and related problems. Advances in Applied Mechanics, 30, $259-445$.

Kachanov, M., Prioul, R., \& Jocker, J. (2010). Incremental linear-elastic response of rocks containing multiple rough fractures: Similarities and differences with traction-free cracks. Geophysics, 75(1), D1-D11. https://doi.org/10.1190/1.3268034

Kachanov, M., \& Sevostianov, I. (2013). Effective properties of heterogeneous materials. Springer Science \& Business Media.

Kachanov, M. L. (1982). A microcrack model of rock inelasticity part I: Frictional sliding on microcracks. Mechanics of Materials, 1(1), 19-27. https://doi.org/10.1016/0167-6636(82)90021-7

Kirkpatrick, S. (1973). Percolation and conduction. Reviews of Modern Physics, 45(4), 574-588. https://doi.org/10.1103/RevModPhys.45.574

Long, J. C. S., Remer, J. S., Wilson, C. R., \& Witherspoon, P. A. (1982). Porous media equivalents for networks of discontinuous fractures. Water Resources Research, 18(3), 645-658. https://doi.org/10.1029/WR018i003p00645

Maillot, J., Davy, P., Goc, R. L., Darcel, C., \& Dreuzy, J. R. d. (2016). Connectivity, permeability, and channeling in randomly distributed and kinematically defined discrete fracture network models. Water Resources Research, 52, 8526-8545. https://doi.org/10.1002/2016WR018973

Nakaya, S., \& Nakamura, K. (2007). Percolation conditions in fractured hard rocks: A numerical approach using the three-dimensional binary fractal fracture network (3D-BFFN) model. Journal of Geophysical Research, 112, B12203. https://doi.org/10.1029/2006JB004670

O'Connell, R. J., \& Budiansky, B. (1974). Seismic velocities in dry and saturated cracked solids. Journal of Geophysical Research, 79(35), 5412-5426. https://doi.org/10.1029/JB079i035p05412

Oda, M. (1985). Permeability tensor for discontinuous rock masses. Géotechnique, 35(4), 483-495. https://doi.org/10.1680/geot.1985.35.4.483 Odling, N. E. (1997). Scaling and connectivity of joint systems in sandstones from western Norway. Journal of Structural Geology, 19(10), 1257-1271. https://doi.org/10.1016/S0191-8141(97)00041-2

Painter, S., \& Cvetkovic, V. (2005). Upscaling discrete fracture network simulations: An alternative to continuum transport models. Water Resources Research, 41, W02002. https://doi.org/10.1029/2004WR003682

Rice, J. R. (1988). Elastic fracture mechanics concepts for interfacial cracks. Journal of Applied Mechanics, 55(1), 98-103. https://doi.org/ $10.1115 / 1.3173668$

Sayers, C. M., \& Kachanov, M. (1995). Microcrack-induced elastic wave anisotropy of brittle rocks. Journal of Geophysical Research, 100(B3), 4149-4156. https://doi.org/10.1029/94JB03134

Schoenberg, M., \& Sayers, C. M. (1995). Seismic anisotropy of fractured rock. Geophysics, 60(1), 204-211. https://doi.org/10.1190/1.1443748 
Selroos, J.-O., Walker, D. D., Ström, A., Gylling, B., \& Follin, S. (2002). Comparison of alternative modelling approaches for groundwater flow in fractured rock. Journal of Hydrology, 257(1-4), 174-188. https://doi.org/10.1016/S0022-1694(01)00551-0

Sevostianov, I., \& Kachanov, M. (2008a). Contact of rough surfaces: A simple model for elasticity, conductivity and cross-property connections. Journal of the Mechanics and Physics of Solids, 56(4), 1380-1400. https://doi.org/10.1016/j.jmps.2007.09.004

Sevostianov, I., \& Kachanov, M. (2008b). Normal and tangential compliances of interface of rough surfaces with contacts of elliptic shape. International Journal of Solids and Structures, 45(9), 2723-2736. https://doi.org/10.1016/j.ijsolstr.2007.12.024

Sevostianov, I., \& Kachanov, M. (2009). Elasticity-conductivity connections for contacting rough surfaces: An overview. Mechanics of Materials, 41(4), 375-384. https://doi.org/10.1016/j.mechmat.2009.01.002

Simmons, G., \& Brace, W. (1965). Comparison of static and dynamic measurements of compressibility of rocks. Journal of Geophysical Research, 70(22), 5649-5656. https://doi.org/10.1029/JZ070i022p05649

Singh, B. (1973). Continuum characterization of jointed rock masses: Part I-The constitutive equations. Paper presented at Int. J. Rock Mech. Min. Sci., Elsevier.

Sneddon, I. N., \& Lowengrub, M. (1969). Crack problems in the classical theory of elasticity (p. 221). New York: Wiley and sons.

Tchalenko, J. S. (1970). Similarities between shear zones of different magnitudes. Geological Society of America Bulletin, 81(6), 1625-1640. https://doi.org/10.1130/0016-7606(1970)81[1625:sbszod]2.0.co;2

Walsh, J. B. (1965a). The effect of cracks on the compressibility of rock. Journal of Geophysical Research, 70(2), 381-389. https://doi.org/ 10.1029/JZ070i002p00381

Walsh, J. B. (1965b). The effect of cracks in rocks on Poisson's ratio. Journal of Geophysical Research, 70(20), 5249-5257. https://doi.org/ 10.1029/JZ070i020p05249

Walsh, J. B. (1965c). The effect of cracks on the uniaxial elastic compression of rocks. Journal of Geophysical Research, 70(2), 399-411. https:// doi.org/10.1029/JZO70i002p00399

Yoshinaka, R., \& Yamabe, T. (1986). 3. Joint stiffness and the deformation behaviour of discontinuous rock. International Journal of Rock Mechanics and Mining Sciences, 23(1), 19-28. https://doi.org/10.1016/0148-9062(86)91663-3

Yoshinaka, R., Yoshida, J., Shimizu, T., Arai, H., \& Arisaka, S. (1993). Scale effect in shear strength and deformability of rock joints. Paper presented at Scale Effects in Rock Masses, Balkema.

Yoshioka, N., \& Scholz, C. H. (1989a). Elastic properties of contacting surfaces under normal and shear loads: 1. Theory. Journal of Geophysical Research, 94(B12), 17,681-17,690. https://doi.org/10.1029/JB094iB12p17681

Yoshioka, N., \& Scholz, C. H. (1989b). Elastic properties of contacting surfaces under normal and shear loads: 2 . Comparison of theory with experiment. Journal of Geophysical Research, 94(B12), 17,691-17,700. https://doi.org/10.1029/JB094iB12p17691 